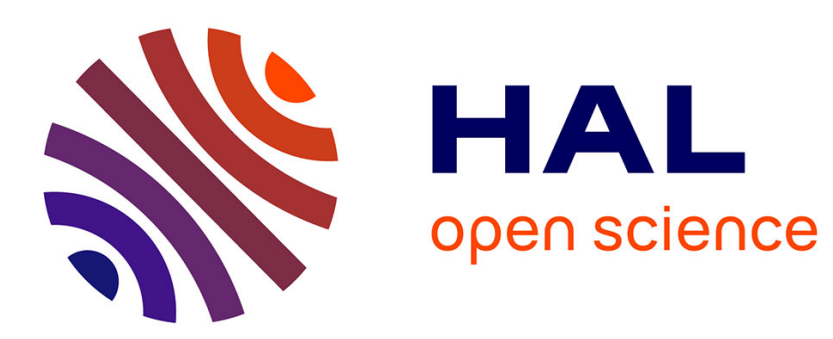

\title{
Global steady-state stabilization and controllability of 1-D semilinear wave equations
}

Jean-Michel Coron, Emmanuel Trélat

\section{To cite this version:}

Jean-Michel Coron, Emmanuel Trélat. Global steady-state stabilization and controllability of 1-D semilinear wave equations. Communications in Contemporary Mathematics, 2006, 8 (4), pp.535-567. hal-00086370

\section{HAL Id: hal-00086370 https://hal.science/hal-00086370}

Submitted on 18 Jul 2006

HAL is a multi-disciplinary open access archive for the deposit and dissemination of scientific research documents, whether they are published or not. The documents may come from teaching and research institutions in France or abroad, or from public or private research centers.
L'archive ouverte pluridisciplinaire HAL, est destinée au dépôt et à la diffusion de documents scientifiques de niveau recherche, publiés ou non, émanant des établissements d'enseignement et de recherche français ou étrangers, des laboratoires publics ou privés. 


\title{
Global steady-state stabilization and controllability of 1-D semilinear wave equations
}

\author{
Jean-Michel Coron*and Emmanuel Trélat ${ }^{\dagger}$
}

\begin{abstract}
This paper is concerned with the exact boundary controllability of semilinear wave equations in one space dimension. We prove that it is possible to move from any steady-state to any other one by means of a boundary control, provided that they are in the same connected component of the set of steady-states. The proof is based on an expansion of the solution in a one-parameter Riesz basis of generalized eigenvectors, and on an effective feedback stabilization procedure which is implemented.
\end{abstract}

MSC classification. Primary: 93B05, 93C20; Secondary: 35B37.

Keywords: Wave equation, stabilization, Riesz basis, pole shifting, Lyapunov functional.

\section{Introduction}

Let $L>0$ fixed and $f: \mathbb{R} \rightarrow \mathbb{R}$ be a function of class $C^{2}$. We are concerned with the exact controllability of the semilinear wave equation

$$
\left\{\begin{array}{l}
\frac{\partial^{2} y}{\partial t^{2}}=\frac{\partial^{2} y}{\partial x^{2}}+f(y) \\
y(t, 0)=0, y_{x}(t, L)=u(t) \\
y(0, \cdot)=a_{0}(\cdot), y_{t}(0, \cdot)=a_{1}(\cdot)
\end{array}\right.
$$

where the state is $\left(y(t, \cdot), y_{t}(t, \cdot)\right):[0, L] \rightarrow \mathbb{R}^{2}$ and the control is $u(t) \in \mathbb{R}$.

The question we investigate in this paper is the following. For $T>0$ large enough, given initial data $\left(a_{0}, a_{1}\right)$ and final data $\left(b_{0}, b_{1}\right)$ in a suitable Hilbert space, is it possible to construct a control $u$ steering the control system $(1)$ from the initial state $\left(y_{0}, y_{1}\right)$ to the target $\left(z_{0}, z_{1}\right)$ within time T? Moreover, is it possible to achieve this by an explicit and efficient numerical implementation?

If $f$ is linear, the situation is well-known (see for instance $[17,21]$ ). In the general semilinear case, the main results as to the global controllability problem, using a variant of the Hilbert Uniqueness Method and a fixed point argument, assert that if $f$ is asymptotically linear (see [25]), and more generally if $f$ is globally Lipschitzian (see [26]), then the control system (1) is globally controllable within time $T>2 L$, in the space $H_{(0)}^{1}(0, L) \times L^{2}(0, L)$, with controls in $L^{2}(0, T)$. The situation extends to slightly superlinear functions, or functions sharing a good sign growth condition, see $[4$, $16,18,25,27]$. Here, and throughout the paper, $H_{(0)}^{1}(0, L)$ denotes the Banach space

$$
H_{(0)}^{1}(0, L):=\left\{y \in H^{1}(0, L) \mid y(0)=0\right\}
$$

\footnotetext{
*Institut Universitaire de France, et Universit Paris-Sud, Math., Equipe AN-EDP, UMR 8628, Bât. 425, 91405 Orsay cedex, France. E-mail: Jean-Michel.Coron@math.u-psud.fr.

†Université Paris-Sud, Math., Equipe AN-EDP, UMR 8628, Bât. 425, 91405 Orsay cedex, France. E-mail: Emmanuel.Trelat@math.u-psud.fr.
} 
endowed with the norm

$$
\|y\|_{H_{(0)}^{1}(0, L)}=\left\|\frac{d y}{d x}\right\|_{L^{2}(0, L)} .
$$

When $f$ is highly superlinear the situation is far more intricate, in particular because of possible blowing up. It is proved in [27] that if there exists $k$ large enough so that

$$
\int_{k}^{+\infty} \frac{d s}{|F(s)|^{1 / 2}}<+\infty
$$

where $F(s)=\int_{0}^{s} f(t) d t$, then the system (1) is not exactly controllable in any time $T>0$. More precisely, for every $T>0$, there exist initial data $\left(a_{0}, a_{1}\right) \in H_{(0)}^{1}(0, L) \times L^{2}(0, L)$ for which the solution of (1) so that $y(0, \cdot)=a_{0}(\cdot)$ and $y_{t}(0, \cdot)=a_{1}(\cdot)$ blows up in time $t<T$, for every control $u \in C^{0}([0, T])$. Hence there is no hope to get a general result on global controllability. The result of this paper is intermediate.

Definition 1. A function $y \in C^{2}([0, L])$ is a steady-state of the control system (1) if

$$
\frac{d^{2} y}{d x^{2}}(x)+f(y(x))=0, y(0)=0 .
$$

Let $\mathcal{S}$ denote the set of steady-states, endowed with the $C^{2}$ topology.

Introduce the Banach space

$$
Y_{T}:=C^{0}\left([0, T], H_{(0)}^{1}(0, L)\right) \cap C^{1}\left([0, T], L^{2}(0, L)\right),
$$

endowed with the norm

$$
\|y\|_{Y_{T}}=\max _{t \in[0, T]}\left(\|y\|_{H_{(0)}^{1}(0, L)}+\left\|\frac{\partial y}{\partial t}\right\|_{L^{2}(0, L)}\right) .
$$

Note that, for every $u \in L^{2}(0, T)$, and for all initial data $\left(a_{0}, a_{1}\right) \in H_{(0)}^{1}(0, L) \times L^{2}(0, L)$, there exists at most one solution of (1) in $Y_{T}$.

The main results of the paper are the following.

Theorem 1. Let $y_{0}$ and $y_{1}$ be two steady-states belonging to a same connected component of $\mathcal{S}$. For every $\delta>0$, there exists $\varepsilon_{1}>0$ so that, for every $\varepsilon \in\left(0, \varepsilon_{1}\right]$, there exists a control $u \in H^{2}(0,1 / \varepsilon)$ such that the solution $y$ in $Y_{1 / \varepsilon}$ of the Cauchy-Dirichlet problem

$$
\left\{\begin{array}{l}
\frac{\partial^{2} y}{\partial t^{2}}=\frac{\partial^{2} y}{\partial x^{2}}+f(y) \\
y(t, 0)=0, y_{x}(t, L)=u(t) \\
y(0, x)=y_{0}(x), y_{t}(0, x)=0
\end{array}\right.
$$

satisfies

$$
\left\|y(1 / \varepsilon, \cdot)-y_{1}(\cdot)\right\|_{H_{(0)}^{1}(0, L)}+\left\|y_{t}(1 / \varepsilon)\right\|_{L^{2}(0, L)} \leq \delta .
$$

Remark 1. The proof of this result, which represents the main part of the paper, relies on an explicit construction of the control $u$ in a feedback form, and of a Lyapunov functional. We stress that the procedure is effective and consists actually in solving a stabilization problem in finite dimension. Indeed in order to construct $u$, one only needs to compute a finite number of quantities related to a one-parameter dependent Riesz expansion of the solution. The numerical procedure is implemented, and simulations are presented in the last section of the paper.

Coupling Theorem 1 with a local controllability result yields the following corollary. 
Corollary 1. Let $y_{0}$ and $y_{1}$ be two steady-states belonging to a same connected component of $\mathcal{S}$. There exist a time $T>0$ and a control function $u \in L^{2}(0, T)$ such that the solution $y(t, x)$ in $Y_{T}$ of the Cauchy-Dirichlet problem (3) satisfies $y(T, \cdot)=y_{1}(\cdot), y_{t}(T, \cdot)=0$.

Remark 2. The time $T$ of controllability required in this result may be large. But on the other part, due to the finite speed of propagation for the wave equation, the time $T$ cannot be arbitrarily small. The question of a minimal time to reach a given target, using for instance a priori estimates, is open.

Remark 3. Similar results have been obtained in [7] in the context of the heat equation. The idea is to stabilize a finite dimensional part of the system using pole shifting. The problem investigated here is however much more challenging, on the one part, because of conservation properties of the wave equation, and on the other, because of the necessity of using Riesz basis expansions. This latter point is the key technical development of this paper, and is investigated in Section 2.3. There is a large body of literature dealing with Riesz basis analysis applied to the boundary controlled wave equation (see for instance $[1,23]$ and references therein). However, the analysis of this article requires a one-parameter Riesz expansion of the solution, so as to obtain a Riesz basis depending smoothly on the parameter (Lemma 5). This reduction procedure constitutes the main contribution of this work.

Remark 4. As proved in [7], the set of steady-states $\mathcal{S}$ is connected if one of the following situations occur:

- $F(y)=\int_{0}^{y} f(s) d s \underset{|y| \rightarrow+\infty}{\longrightarrow}+\infty$;

- for every $\alpha>0$, the indefinite integral

$$
\int \frac{d y}{\sqrt{\alpha-F(y)}}
$$

(if it makes sense) diverges in $-\infty$ and in $+\infty$;

- the function $f$ is odd, i.e. $f(-y)=-f(y)$, for every $y \in \mathbb{R}$.

Remark 5. The result of Corollary 1 may be achieved directly by using repeatedly a local exact controllability theorem (see [25, 27], and Section 2.6 of this paper), but contrarily to our strategy, the control function is not constructed explicitly. Note also that our approach does not necessarily require controllability of the linearized system around an equilibrium (see [6]).

Remark 6. In the case of the heat equation [7], it was proved that, if the steady-states $y_{0}$ and $y_{1}$ belong to distinct connected components of the set $\mathcal{S}$ of steady-states, then it is impossible, either to move from $y_{0}$ to $y_{1}$, or the converse. Here, in the case of the wave equation, the question is open.

The idea of the proof of Theorem 1 is as follows. Linearizing the system (3) along a path of steadystates joining $y_{0}$ to $y_{1}$, we obtain a system of the form $w_{t t}=w_{x x}+c w$, where $c \in L^{\infty}(0, T)$, with boundary conditions $w(t, 0)=0$ and $w_{x}(t, L)=v(t)$. At the first glance, if we suppose that $c=0$, then it is possible to choose a control $v(\cdot)$ stabilizing this equation; namely, if we set $v(t)=-\alpha w_{t}(t, L)$, with $\alpha>0$, the energy function

$$
t \mapsto \int_{0}^{L}\left(w_{t}(t, x)^{2}+w_{x}(t, x)^{2}\right) d t
$$

is exponentially decreasing (see for instance $[13,14]$ for some results in that direction). Moreover, an obvious spectral computation shows that the eigenvalues of the corresponding operator have their real part tending to $-\infty$ as $\alpha$ tends to 1 . This result only holds asymptotically if $c \neq 0$. Therefore, in the general case, if $\alpha$ is close enough to 1 , then only a finite number of eigenvalues may be positive. The system corresponding to these unstable modes can be written (using an expansion of the solution in a one-parameter dependent Riesz basis of generalized eigenvectors), at the first order, as a nonautonomous linear control system. It is then possible, by a pole shifting procedure together with a time reparametrization, to stabilize this subsystem using a control in a feedback form. 
Remark 7. The method consisting in stabilizing a quasi-static deformation has already been used in [7] in the context of nonlinear heat equations, in [22] for Navier-Stokes equations, in [6] for shallow water equations, and in [3] for a Schrödinger equation. However, in both latter cases, the deformation was naturally stable and a feedback procedure was not necessary.

\section{Proof of the main results}

\subsection{Construction of a path of steady-states}

The following lemma is obvious.

Lemma 1. Let $\phi_{0}, \phi_{1} \in \mathcal{S}$. Then $\phi_{0}$ and $\phi_{1}$ belong to the same connected component of $\mathcal{S}$ if and only if, for every real number $\alpha$ between $\phi_{0}^{\prime}(0)$ and $\phi_{1}^{\prime}(0)$, the maximal solution of

$$
\frac{d^{2} y}{d x^{2}}+f(y)=0, y(0)=0, y^{\prime}(0)=\alpha
$$

denoted by $y^{\alpha}(\cdot)$, is defined on $[0, L]$.

Let now $y_{0}$ and $y_{1}$ in the same connected component of $\mathcal{S}$. Let us construct in $\mathcal{S}$ a $C^{2}$ path $(\hat{y}(\tau, \cdot), \hat{u}(\tau)), 0 \leq \tau \leq 1$, joining $y_{0}$ to $y_{1}$. For $i=0,1$, set $\alpha_{i}:=y_{i}^{\prime}(0)$. Then, with our previous notations, one has $y_{i}(\cdot)=y^{\alpha_{i}}(\cdot), i=0,1$. Now set

$$
\hat{y}(\tau, x):=y^{(1-\tau) \alpha_{0}+\tau \alpha_{1}}(x) \text { and } \hat{u}(\tau):=\hat{y}_{x}(\tau, L),
$$

where $\tau \in[0,1]$ and $x \in[0, L]$, so that $\hat{y}(\tau, \cdot)$ satisfies

$$
\left\{\begin{array}{l}
\frac{\partial^{2} \hat{y}}{\partial x^{2}}(\tau, x)+f(\hat{y}(\tau, x))=0, x \in(0, L) \\
\hat{y}(\tau, 0)=0, \frac{\partial \hat{y}}{\partial x}(\tau, 0)=(1-\tau) \alpha_{0}+\tau \alpha_{1}
\end{array}\right.
$$

for all $\tau \in[0,1]$. By construction we have

$$
\hat{y}(0, \cdot)=y_{0}(\cdot) \text { and } \hat{y}(1, \cdot)=y_{1}(\cdot),
$$

and thus $(\hat{y}(\tau, \cdot), \hat{u}(\tau))$ is a $C^{2}$ path in $\mathcal{S}$ connecting $y_{0}$ to $y_{1}$.

\subsection{Reduction of the problem}

Let $\varepsilon>0$, and let $y$ denote the solution of (3) in $Y_{1 / \varepsilon}$, associated to a control $u \in H^{2}(0,1 / \varepsilon)$. We set, for all $t \in[0,1 / \varepsilon]$ and $x \in[0, L]$,

$$
\begin{aligned}
z(t, x) & :=y(t, x)-\hat{y}(\varepsilon t, x), \\
u_{1}(t) & :=u(t)-\hat{u}(\varepsilon t) .
\end{aligned}
$$

This time reparametrization will happen to be useful in order to perform a pole shifting procedure on the linear finite dimensional system representing the unstable part of the equation.

From the definition of $(\hat{y}, \hat{u})$ we infer that $z$ satisfies the initial-boundary problem

$$
\left\{\begin{array}{l}
z_{t t}=z_{x x}+f^{\prime}(\hat{y}) z+z^{2} \int_{0}^{1}(1-s) f^{\prime \prime}(\hat{y}+s z) d s-\varepsilon^{2} \hat{y}_{\tau \tau} \\
z(t, 0)=0, z_{x}(t, L)=u_{1}(t) \\
z(0, x)=0, z_{t}(0, x)=-\varepsilon \hat{y}_{\tau}(0, x)
\end{array}\right.
$$


Notice that, if the nonlinearity $f$ and the residual term $r$ were equal to zero, then, as explained previously, setting $u_{1}(t)=-\alpha z_{t}(t, L)$, the energy function

$$
t \mapsto \int_{0}^{L}\left(z_{t}(t, x)^{2}+z_{x}(t, x)^{2}\right) d t
$$

would be exponentially decreasing. This suggests to seek the control function $u_{1}(t)$ in the form

$$
u_{1}(t)=-\alpha z_{t}(t, L)+v(t)
$$

where $\alpha>0$ will be chosen later. Set

$$
w(t, x):=z(t, x)-\frac{x(x-L)}{L} v(t)
$$

This leads to the system

$$
\left\{\begin{array}{l}
w_{t t}=w_{x x}+f^{\prime}(\hat{y}) w-\frac{x(x-L)}{L} v^{\prime \prime}+\left(\frac{x(x-L)}{L} f^{\prime}(\hat{y})+\frac{2}{L}\right) v+r(\varepsilon, t, x), \\
w(t, 0)=0, w_{x}(t, L)=-\alpha w_{t}(t, L) \\
w(0, x)=-\frac{x(x-L)}{L} v(0), w_{t}(0, x)=-\varepsilon \hat{y}_{\tau}(0, x)-\frac{x(x-L)}{L} v^{\prime}(0)
\end{array}\right.
$$

where

$$
r(\varepsilon, t, x)=\left(w+\frac{x(x-L)}{L} v\right)^{2} \int_{0}^{1}(1-s) f^{\prime \prime}\left(\hat{y}+s\left(w+\frac{x(x-L)}{L} v\right)\right) d s-\varepsilon^{2} \hat{y}_{\tau \tau} .
$$

The aim is to prove that, given a neighborhood $\mathcal{V}$ of $(0,0,0,0)$ in $\mathbb{R} \times \mathbb{R} \times \mathrm{H}_{(0)}^{1}(0, \mathrm{~L}) \times \mathrm{L}^{2}(0, \mathrm{~L})$, for $\varepsilon>0$ small enough, there exists a pair $(v, w)$ solution of $(7)$, satisfying $v(0)=v^{\prime}(0)=0$, such that

$$
\left(v(1 / \varepsilon), v^{\prime}(1 / \varepsilon), w(1 / \varepsilon, \cdot), w_{t}(1 / \varepsilon, \cdot)\right) \in \mathcal{V} .
$$

To achieve this, we shall construct an appropriate control function and a Lyapunov functional which stabilizes system (7) to 0 .

Remark 8. Let us set an upper bound to the residual term $r$. First, it is not difficult to check that there exists a constant $C_{1}$ such that, if $|v(t)|+\|w(t, .)\|_{L^{\infty}(0, L)} \leq 1$, then the inequality

$$
\|r(\varepsilon, t, \cdot)\|_{L^{\infty}(0, L)} \leq C_{1}\left(\varepsilon^{2}+v(t)^{2}+\|w(t, \cdot)\|_{L^{\infty}(0, L)}^{2}\right)
$$

holds. Moreover, since $w(t, 0)=0$, we can assert that there exists a constant $C_{2}$ such that, if $|v(t)|+\|w(t, \cdot)\|_{L^{\infty}(0, L)} \leq 1$, then

$$
\|r(\varepsilon, t, \cdot)\|_{L^{\infty}(0, L)} \leq C_{2}\left(\varepsilon^{2}+v(t)^{2}+\left\|w_{x}(t, \cdot)\right\|_{L^{2}(0, L)}^{2}\right) .
$$

This a priori estimate shall be used later.

\subsection{Asymptotic Riesz spectral analysis of the operator}

The proof is based on a spectral analysis of the operator representing the system (7). In what follows, we set

$$
H:=\left\{\left(\begin{array}{l}
w^{1} \\
w^{2}
\end{array}\right) \in H^{1}((0, L), \mathbb{C}) \times L^{2}((0, L), \mathbb{C}) \mid w^{1}(0)=0\right\} .
$$


Endowed with the scalar product

$$
\left\langle\left(\begin{array}{l}
w^{1} \\
w^{2}
\end{array}\right),\left(\begin{array}{l}
z^{1} \\
z^{2}
\end{array}\right)\right\rangle_{H}:=\int_{0}^{L}\left(\overline{w_{x}^{1}} z_{x}^{1}+\overline{w^{2}} z^{2}\right) d x,
$$

where the overbar denotes the complex conjugate, $H$ is a complex Hilbertian space.

It is relevant to write (7) in the form

$$
\left\{\begin{array}{l}
w_{t}^{1}=w^{2} \\
w_{t}^{2}=w_{x x}^{1}+f^{\prime}(\hat{y}) w^{1}-\frac{x(x-L)}{L} v^{\prime \prime}+\left(\frac{x(x-L)}{L} f^{\prime}(\hat{y})+\frac{2}{L}\right) v+r(\varepsilon, t, x), \\
w^{1}(t, 0)=0, w_{x}^{1}(t, L)=-\alpha w^{2}(t, L) \\
w^{1}(0, x)=-\frac{x}{L} v(0), w^{2}(0, x)=-\varepsilon \hat{y}_{\tau}(0, x)-\frac{x(x-L)}{L} v^{\prime}(0),
\end{array}\right.
$$

and to introduce the one-parameter family of linear operators

$$
\widetilde{A}(\tau):=\left(\begin{array}{cc}
0 & 1 \\
A(\tau) & 0
\end{array}\right)
$$

where $A(\tau):=\triangle+f^{\prime}(\hat{y}(\tau, \cdot)) \operatorname{Id}, \tau \in[0,1]$, on the domain

$$
\begin{gathered}
D(\widetilde{A}(\tau)):=\left\{\left(\begin{array}{l}
w^{1} \\
w^{2}
\end{array}\right) \in H \mid w^{1} \in H^{2}((0, L), \mathbb{C}), w^{2} \in H^{1}((0, L), \mathbb{C}),\right. \\
\left.w^{2}(0)=0, w_{x}^{1}(L)=-\alpha w^{2}(L)\right\},
\end{gathered}
$$

so that

$$
W_{t}(t, x)=\widetilde{A}(\varepsilon t) W(t, x)+v(t) a(\varepsilon t, x)+v^{\prime \prime}(t) b(x)+R(\varepsilon, t, x),
$$

where

$$
\begin{aligned}
a(\tau, x) & :=\left(\begin{array}{c}
0 \\
\frac{x(x-L)}{L} f^{\prime}(\hat{y}(\tau, x))+\frac{2}{L}
\end{array}\right)=:\left(\begin{array}{l}
a^{1}(\tau, x) \\
a^{2}(\tau, x)
\end{array}\right), \\
b(x) & :=\left(\begin{array}{c}
0 \\
-\frac{x(x-L)}{L}
\end{array}\right)=:\left(\begin{array}{l}
b^{1}(x) \\
b^{2}(x)
\end{array}\right), \\
W(t, x) & :=\left(\begin{array}{c}
w^{1}(t, x) \\
w^{2}(t, x)
\end{array}\right), R(\varepsilon, t, x):=\left(\begin{array}{c}
0 \\
r(\varepsilon, t, x)
\end{array}\right) .
\end{aligned}
$$

Recall that, by definition, the sequence $\left(\psi_{j}\right)_{j \in \mathbb{Z}}$ is a Riesz basis of the Hilbert space $H$ if and only if there exists an equivalent scalar product on $H$ for which $\left(\psi_{j}\right)_{j \in \mathbb{Z}}$ is orthonormal (see [9]); this is equivalent to the existence of positive constants $A, B$ such that, for every sequence of complex scalars $\left(c_{j}\right)_{j \in \mathbb{Z}}$, there holds

$$
A \sum_{j \in \mathbb{Z}}\left|c_{j}\right|^{2} \leq\left\|\sum_{j \in \mathbb{Z}} c_{j} \psi_{j}\right\|_{H}^{2} \leq B \sum_{j \in \mathbb{Z}}\left|c_{j}\right|^{2} .
$$

An operator $A$ on $H$ is said to have compact resolvent whenever there exists a real $\alpha$ in the resolvent of $A$ so that $(\alpha \mathrm{Id}-A)^{-1}$ is compact in $H$.

A nontrivial element $v \in H$ is called a generalized eigenvector of $A$ (resp., an eigenvector of $A$ ), associated to the eigenvalue $\lambda$, if there exists a positive integer $n$ so that $(\lambda I d-A)^{n} v=0$ (resp., if $(\lambda \mathrm{Id}-A) v=0)$. The algebraic multiplicity (resp., the geometric multiplicity) of $\lambda$ is defined as the number of linearly independent generalized eigenvectors (resp., eigenvectors) associated to $\lambda$.

Recall that the spectrum of operator $A$ on $H$ having compact resolvent consists of isolated eigenvalues only, and each eigenvalue has finite algebraic multiplicity. 
Lemma 2. For every $\tau \in[0,1]$, the operator $\widetilde{A}(\tau)$ in $H$ has compact resolvent, and thus its spectrum consists of isolated eigenvalues. There exists a Riesz basis $\left(\tilde{e}_{k}(\tau, \cdot)\right)_{k \in \mathbb{Z}}$ of $H$, consisting of generalized eigenfunctions of $\widetilde{A}(\tau)$, associated to the eigenvalues $\left(\lambda_{k}(\tau)\right)_{k \in \mathbb{Z}}$, such that:

(i) $\tilde{e}_{k}(\tau, \cdot) \in D(\widetilde{A}(\tau))$, and $\left\|\tilde{e}_{k}(\tau, \cdot)\right\|_{H}=1$, for every $k \in \mathbb{Z}$ and every $\tau \in[0,1]$;

(ii) each eigenvalue $\lambda_{k}(\tau)$ is geometrically simple;

(iii) there exists an integer $n_{0} \geq 0$ so that, for every integer $k$ satisfying $|k|>n_{0}$, the eigenvalue $\lambda_{k}(\tau)$ is algebraically simple, and satisfies

$$
\lambda_{k}(\tau)=\frac{1}{2 L} \ln \frac{\alpha-1}{\alpha+1}+i \frac{k \pi}{L}+\mathrm{O}\left(\frac{1}{|k|}\right)
$$

as $|k| \rightarrow+\infty$, uniformly for $\tau \in[0,1]$;

(iv) if $|k|>n_{0}$, then the generalized eigenfunction $\tilde{e}_{k}(\tau, \cdot)$ is an eigenfunction of $\widetilde{A}(\tau)$, associated to the (algebraically simple) eigenvalue $\lambda_{k}(\tau)$, and the functions

$$
\begin{aligned}
{[0,1] } & \rightarrow \mathbb{C} \\
\tau & \mapsto \lambda_{k}(\tau),
\end{aligned}
$$

and

$$
\begin{aligned}
{[0,1] } & \rightarrow H \\
\tau & \mapsto \tilde{e}_{k}(\tau, \cdot)
\end{aligned}
$$

are of class $C^{1}$.

(v) for every integer $k>n_{0}$ and every $\tau \in[0,1]$,

$$
\lambda_{k}(\tau)=\overline{\lambda_{-k}(\tau)} \text {, and } \tilde{e}_{k}(\tau, \cdot)=\overline{\tilde{e}_{-k}(\tau, \cdot)} .
$$

Moreover, the Riesz basis $\left(\tilde{e}_{k}(\tau, \cdot)\right)_{k \in \mathbb{Z}}$ of $H$ is uniform with respect to $\tau \in[0,1]$, in the sense that

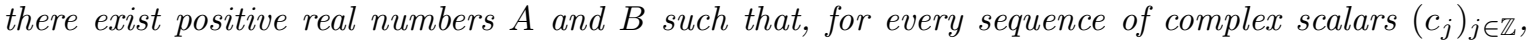
there holds

$$
A \sum_{j \in \mathbb{Z}}\left|c_{j}\right|^{2} \leq\left\|\sum_{j \in \mathbb{Z}} c_{j} \tilde{e}_{j}(\tau, \cdot)\right\|_{H}^{2} \leq B \sum_{j \in \mathbb{Z}}\left|c_{j}\right|^{2} .
$$

Remark 9. Uniform Riesz property (20) would be obvious if all eigenfunctions $\tilde{e}_{k}$ were continuous with respect to $\tau$. However, the function $\tau \mapsto e_{k}(\tau, \cdot)$ may fail to be continuous whenever $|k| \leq n_{0}$, due to the fact that the eigenvalue $\lambda_{k}(\tau)$ is not necessarily algebraically simple.

Proof of Lemma 2. The fact that the operator $\widetilde{A}(\tau)$ has compact resolvent on $H$ is obvious. For $\tau \in[0,1]$, let $\left(\begin{array}{l}w^{1} \\ w^{2}\end{array}\right)$ be an eigenfunction of $\widetilde{A}(\tau)$ associated to the eigenvalue $\lambda$. Then,

$$
\begin{aligned}
& w^{2}=\lambda w^{1}, A(\tau) w^{1}=\lambda w^{2} \\
& w^{1}(0)=w^{2}(0)=0, w_{x}^{1}(L)=-\alpha w^{2}(L) .
\end{aligned}
$$

Therefore, $w^{1}$ satisfies the boundary value problem

$$
\left\{\begin{array}{l}
w_{x x}^{1}+f^{\prime}(\hat{y}) w^{1}=\lambda^{2} w^{1}, \\
w^{1}(0)=0, w_{x}^{1}(L)=-\lambda \alpha w^{1}(L) .
\end{array}\right.
$$

If we assume that $|\lambda|$ tends to $+\infty$, then it is not difficult to show that, for every $x \in[0, L]$,

$$
w^{1}(x)=\sinh \sqrt{\lambda^{2}+\mathrm{O}(1)} x \text {, and } w_{x}^{1}(x)=\sqrt{\lambda^{2}+\mathrm{O}(1)} \cosh \sqrt{\lambda^{2}+\mathrm{O}(1)} x,
$$


as $|\lambda| \rightarrow+\infty$, uniformly with respect to $\tau \in[0,1]$ and $x \in[0, L]$. If we seek $\lambda$ in the form $\lambda=-\theta+i \nu$, with $\nu$ large enough, then easy computations show that there exists an integer $k$ so that, as $|k| \rightarrow+\infty$,

$$
\nu=\frac{k \pi}{L}+\mathrm{O}\left(\frac{1}{|k|}\right), \text { and } \frac{\alpha-1}{\alpha+1} \mathrm{e}^{2 \theta L}=1,
$$

and thus,

$$
\lambda_{k}(\tau)=\frac{1}{2 L} \ln \frac{\alpha-1}{\alpha+1}+i \frac{k \pi}{L}+\mathrm{O}\left(\frac{1}{|k|}\right)
$$

Let us prove that each eigenvalue $\lambda_{k}(\tau)$ is geometrically simple. If not, let $\left(\begin{array}{l}w_{1}^{1} \\ w_{1}^{2}\end{array}\right)$ and $\left(\begin{array}{l}w_{2}^{1} \\ w_{2}^{2}\end{array}\right)$ be two independent eigenfunctions associated to the eigenvalue $\lambda$. Let us first point out that $w_{1}^{1}(L) \neq 0$. Indeed, if $w_{1}^{1}(L)=0$, then $w_{1}^{1}$ satisfies

$$
\left\{\begin{array}{l}
w_{1 x x}^{1}+f^{\prime}(\hat{y}) w_{1}^{1}=\lambda^{2} w_{1}^{1}, \\
w_{1}^{1}(L)=w_{1 x}^{1}(L)=0,
\end{array}\right.
$$

and thus $w_{1}^{1} \equiv 0$. Since $w_{1}^{2}=\lambda w_{1}^{1}$, one gets $\left(w_{1}^{1}, w_{1}^{2}\right) \equiv(0,0)$, which is a contradiction. If we set $w=w_{2}^{1}(L) w_{1}^{1}-w_{1}^{1}(L) w_{2}^{1}$, then $w$ satisfies

$$
\left\{\begin{array}{l}
w_{x x}+f^{\prime}(\hat{y}) w=\lambda^{2} w, \\
w(L)=w_{x}(L)=0,
\end{array}\right.
$$

and thus $w \equiv 0$, whence $\left(\begin{array}{l}w_{1}^{1} \\ w_{1}^{2}\end{array}\right)$ and $\left(\begin{array}{l}w_{2}^{1} \\ w_{2}^{2}\end{array}\right)$ are not linearly independent, which is a contradiction. Hence, the item (ii) of the lemma follows.

Let $\left(\tilde{e}_{k}(\tau, \cdot)\right)_{k \in \mathbb{Z}}$ denote a complete set of generalized eigenfunctions of $\widetilde{A}(\tau)$, associated to the eigenvalues $\left(\lambda_{k}(\tau)\right)_{k \in \mathbb{Z}}$, and such that the item (i) of the lemma holds. In order to prove that $\left(\tilde{e}_{k}(\tau, \cdot)\right)_{k \in \mathbb{Z}}$ is a Riesz basis of $H$, we use Bari's Theorem (see for instance [9, Theorem 2.3 p. 317], see also [10, Theorem 6.3]). From this result, if we are able to exhibit a Riesz basis $\left(\phi_{k}\right)_{k \in \mathbb{Z}}$ of $H$ which is quadratically close to $\left(\tilde{e}_{k}(\tau, \cdot)\right)_{k \in \mathbb{Z}}$, that is,

$$
\sum_{k}\left\|\phi_{k}(\cdot)-\tilde{e}_{k}(\tau, \cdot)\right\|_{H}^{2}<\infty
$$

then the sequence $\left(\tilde{e}_{k}(\tau, \cdot)\right)_{k \in \mathbb{Z}}$ is a Riesz basis of $H$.

To this aim, we introduce in $H$ the operator

$$
\tilde{A}_{0}:=\left(\begin{array}{rr}
0 & 1 \\
\triangle & 0
\end{array}\right)
$$

on the same domain (14) than $\widetilde{A}(\tau)$. Intuitively, this operator corresponds to a truncation of $\widetilde{A}(\tau)$, up to the compact part $f^{\prime}(\hat{y}(\tau, \cdot))$ Id. Bari's theorem, and simple computations, all of them detailed in $[21$, Section 4 , p. 667$]$ show that the operator $\tilde{A}_{0}$ admits a Riesz basis of eigenfunctions $\left(\phi_{k}\right)_{k \in \mathbb{Z}}$, associated to the eigenvalues $\left(\mu_{k}\right)_{k \in \mathbb{Z}}$, so that there holds, for every integer $k$,

$$
\mu_{k}=\frac{1}{2 L} \ln \frac{\alpha-1}{\alpha+1}+i \frac{k \pi}{L}
$$

and

$$
\phi_{k}=\left(\begin{array}{c}
\phi_{k}^{1} \\
\phi_{k}^{2}
\end{array}\right)
$$

where

$$
\phi_{k}^{1}(x)=\frac{1}{A_{k}} \sinh \mu_{k} x, \phi_{k}^{2}(x)=\frac{\mu_{k}}{A_{k}} \cosh \mu_{k} x,
$$


with

$$
A_{k}=\frac{1}{2 L \sqrt{-\operatorname{Re}\left(\mu_{k}\right)}} \sqrt{\left(\mathrm{e}^{-2 \operatorname{Re}\left(\mu_{k}\right) L}-\mathrm{e}^{2 \operatorname{Re}\left(\mu_{k}\right) L}\right)\left(k^{2} \pi^{2}+\left(\operatorname{Re}\left(\mu_{k}\right)\right)^{2} L^{2}\right)} .
$$

Moreover, the eigenvalues $\mu_{k}$ are algebraically simple as $|k| \rightarrow+\infty$. From expansions (18) and (21), we get easily, in $H$,

$$
\tilde{e}_{k}(\tau, \cdot)=\phi_{k}(\cdot)+\mathrm{O}(1 / k),
$$

uniformly for $\tau \in[0,1]$. Hence, the family $\left(\phi_{k}\right)_{k \in \mathbb{Z}}$ is quadratically close to $\left(\tilde{e}_{k}(\tau, \cdot)\right)_{k \in \mathbb{Z}}$, uniformly for $\tau \in[0,1]$. The proof of Bari's Theorem in [9, Theorem 2.3 p. 317] readily extends to our case, and and the uniform Riesz property (20) follows. Moreover, the eigenvalues $\lambda_{k}(\tau)$ are algebraically simple as $|k| \rightarrow+\infty$.

In particular, with the formula (22), the item (iii) follows.

Moreover, it is a standard fact that, if $|k|>n_{0}$, then the eigenfunction $\tilde{e}_{k}(\tau, \cdot)$ and the eigenvalue $\lambda_{k}(\tau)$ are $C^{1}$ functions of $\tau$ (see for instance [11, 20]). The item (iv) is proved.

Finally, note that it is possible to choose the eigenelements so that item (v) holds. Indeed, one just has to show that the operator $\widetilde{A}(\tau)$ admits (at least) a real eigenvalue. But this follows obviously from an homotopy argument using the operator $\tilde{A}_{0}$.

Let $\widetilde{A}(\tau)^{*}$ denote the adjoint operator of $\widetilde{A}(\tau)$ on $H$. The following lemma is obvious.

Lemma 3. For every $\tau \in[0,1]$, the domain of $\widetilde{A}(\tau)^{*}$ is given by

$$
\begin{gathered}
D\left(\widetilde{A}(\tau)^{*}\right)=\left\{\left(\begin{array}{l}
z^{1} \\
z^{2}
\end{array}\right) \in H \mid z^{1} \in H^{2}((0, L), \mathbb{C}), z^{2} \in H^{1}((0, L), \mathbb{C}),\right. \\
\left.z^{2}(0)=0, z_{x}^{1}(L)=\alpha z^{2}(L)\right\} .
\end{gathered}
$$

For every $\left(\begin{array}{c}z^{1} \\ z^{2}\end{array}\right) \in D\left(\widetilde{A}(\tau)^{*}\right)$, there holds

$$
\widetilde{A}(\tau)^{*}\left(\begin{array}{c}
z^{1} \\
z^{2}
\end{array}\right)=-\left(\begin{array}{c}
z^{2}+g \\
z_{x x}^{1}
\end{array}\right),
$$

where the function $g \in C^{2}([0, L], \mathbb{C})$ is defined by

$$
\left\{\begin{array}{l}
g_{x x}=f^{\prime}(\hat{y}(\tau, \cdot)) z^{2} \\
g(0)=g_{x}(L)=0
\end{array}\right.
$$

We next introduce the dual Riesz basis $\left(\tilde{f}_{j}(\tau, \cdot)\right)_{j \in \mathbb{Z}}$ of $\left(\tilde{e}_{j}(\tau, \cdot)\right)_{j \in \mathbb{Z}}$. Recall that, by definition, there holds

$$
\left\langle\tilde{f}_{j}(\tau, \cdot), \tilde{e}_{k}(\tau, \cdot)\right\rangle_{H}=\delta_{k j}=\left\{\begin{array}{l}
1 \text { if } k=j, \\
0 \text { otherwise }
\end{array}\right.
$$

and moreover $\left(\tilde{f}_{j}(\tau, \cdot)\right)_{j \in \mathbb{Z}}$ is a Riesz basis on $H$ of generalized eigenvectors of $\widetilde{A}(\tau)^{*}$ with associated eigenvalues $\left(\overline{\lambda_{j}(\tau)}\right)_{j \in \mathbb{Z}}$.

Remark 10. Reasoning as in the proof of Lemma 2, we state that $\left(\tilde{f}_{j}(\tau, \cdot)\right)_{j \in \mathbb{Z}}$ is a uniform Riesz basis on $H$, in the sense that there exist positive real numbers $A^{\prime}$ and $B^{\prime}$ such that, for every sequence of complex scalars $\left(c_{j}\right)_{j \in \mathbb{Z}}$, there holds

$$
A^{\prime} \sum_{j \in \mathbb{Z}}\left|c_{j}\right|^{2} \leq\left\|\sum_{j \in \mathbb{Z}} c_{j} \tilde{f}_{j}(\tau, \cdot)\right\|_{H}^{2} \leq B^{\prime} \sum_{j \in \mathbb{Z}}\left|c_{j}\right|^{2}
$$

In particular, the sequence of real numbers $\left(\left\|\tilde{f}_{j}(\tau, \cdot)\right\|_{H}\right)_{j \in \mathbb{Z}}$ is bounded, uniformly for $\tau \in[0,1]$. 
In the sequel, we will need the following technical lemma.

Lemma 4. There exists a constant $C>0$ so that

$$
\left\|\tilde{e}_{k \tau}(\tau, \cdot)\right\|_{H} \leq \frac{C}{|k|}, \text { and }\left\|\tilde{f}_{k \tau}(\tau, \cdot)\right\|_{H} \leq \frac{C}{|k|},
$$

for every integer $k$ satisfying $|k|>n_{0}$, and every $\tau \in[0,1]$.

Proof. Let $k$ be an integer such that $|k|>n_{0}$. From Lemma 2, the eigenfunction $\tilde{e}_{k}(\tau, \cdot)$ is a $C^{1}$ function of $\tau$. We consider the expansion of $\tilde{e}_{k \tau}(\tau, \cdot)$ as series in the Riesz basis $\left(\tilde{e}_{j}(\tau, \cdot)\right)_{j \in \mathbb{Z}}$, convergent in $H$,

$$
\tilde{e}_{k \tau}(\tau, \cdot)=\sum_{j \in \mathbb{Z}} \alpha_{j}^{k}(\tau) \tilde{e}_{j}(\tau, \cdot)
$$

where $\alpha_{j}^{k}(\tau)$ is defined by

$$
\alpha_{j}^{k}(\tau):=\left\langle\tilde{f}_{j}(\tau, \cdot), \tilde{e}_{k \tau}(\tau, \cdot)\right\rangle_{H},
$$

for every $\tau \in[0,1]$, and all $j, k \in \mathbb{Z}$, with $|k|>n_{0}$.

Let us estimate $\alpha_{j}^{k}(\tau)$, for large values of $|j|$ and $|k|$. By definition of $\tilde{e}_{k}(\tau, \cdot)$, and from Lemma 2, we have, whenever $|k|>n_{0}$,

$$
\widetilde{A}(\tau) \tilde{e}_{k}(\tau, \cdot)=\lambda_{k}(\tau) \tilde{e}_{k}(\tau, \cdot)
$$

Since the domain of $\widetilde{A}(\tau)$ does not depend on $\tau$, it is clear that $\tilde{e}_{k \tau}(\tau, \cdot) \in D(\widetilde{A}(\tau))$. Differentiating with respect to $\tau$, we get

$$
\widetilde{A}(\tau) \tilde{e}_{k \tau}(\tau, \cdot)=\lambda_{k}^{\prime}(\tau) \tilde{e}_{k}(\tau, \cdot)+\lambda_{k}(\tau) \tilde{e}_{k \tau}(\tau, \cdot)-\widetilde{A}^{\prime}(\tau) \tilde{e}_{k}(\tau, \cdot),
$$

and thus, taking the scalar product with $f_{j}(\tau, \cdot), j \in \mathbb{Z}$, we get

$$
\begin{aligned}
\left\langle\widetilde{A}(\tau)^{*} \tilde{f}_{j}(\tau, \cdot), \tilde{e}_{k \tau}(\tau, \cdot)\right\rangle_{H} & =\left\langle\tilde{f}_{j}(\tau, \cdot), \widetilde{A}(\tau) \tilde{e}_{k \tau}(\tau, \cdot)\right\rangle_{H} \\
& =\lambda_{k}^{\prime}(\tau) \delta_{k j}+\lambda_{k}(\tau) \alpha_{j}^{k}(\tau)-\left\langle\tilde{f}_{j}(\tau, \cdot), \widetilde{A}^{\prime}(\tau) \tilde{e}_{k}(\tau, \cdot)\right\rangle_{H} .
\end{aligned}
$$

We distinguish between two cases.

First case. If $|j|>n_{0}$, then $\widetilde{A}(\tau)^{*} \tilde{f}_{j}(\tau, \cdot)=\overline{\lambda_{j}(\tau)} \tilde{f}_{j}(\tau, \cdot)$, and thus (27) yields, for $j \neq k$,

$$
\alpha_{j}^{k}(\tau) \lambda_{j}(\tau)=\lambda_{k}(\tau) \alpha_{j}^{k}(\tau)-\left\langle\tilde{f}_{j}(\tau, \cdot), \widetilde{A}^{\prime}(\tau) \tilde{e}_{k}(\tau, \cdot)\right\rangle_{H},
$$

and for $j=k$,

$$
\lambda_{k}^{\prime}(\tau)=\left\langle\tilde{f}_{k}(\tau, \cdot), \widetilde{A}^{\prime}(\tau) \tilde{e}_{k}(\tau, \cdot)\right\rangle_{H} .
$$

Since $\lambda_{j}(\tau) \neq \lambda_{k}(\tau)$ whenever $j \neq k,|j|>n_{0},|k|>n_{0}$, there holds

$$
\alpha_{j}^{k}(\tau)=\frac{1}{\lambda_{k}(\tau)-\lambda_{j}(\tau)}\left\langle\tilde{f}_{j}(\tau, \cdot), \widetilde{A}^{\prime}(\tau) \tilde{e}_{k}(\tau, \cdot)\right\rangle_{H} .
$$

Clearly,

and thus, denoting

$$
\widetilde{A}^{\prime}(\tau)=\left(\begin{array}{cc}
0 & 0 \\
f^{\prime \prime}(\hat{y}(\tau, \cdot)) \hat{y}_{\tau}(\tau, \cdot) & 0
\end{array}\right)
$$

$$
\tilde{e}_{k}(\tau, \cdot)=\left(\begin{array}{c}
\tilde{e}_{k}^{1}(\tau, \cdot) \\
\tilde{e}_{k}^{2}(\tau, \cdot)
\end{array}\right), \text { and } \tilde{f}_{j}(\tau, \cdot)=\left(\begin{array}{c}
\tilde{f}_{j}^{1}(\tau, \cdot) \\
\left.\tilde{f}_{j}^{2}(\tau, \cdot)\right)
\end{array}\right)
$$

we get

$$
\left\langle\tilde{f}_{j}(\tau, \cdot), \widetilde{A}^{\prime}(\tau) \tilde{e}_{k}(\tau, \cdot)\right\rangle_{H}=\int_{0}^{L} f^{\prime \prime}(\hat{y}(\tau, x)) \hat{y}_{\tau}(\tau, x) \tilde{e}_{k}^{1}(\tau, x) \overline{\tilde{f}_{j}^{2}(\tau, x)} d x
$$


Since $\tilde{e}_{k}(\tau, \cdot)$ is an eigenfunction of $\widetilde{A}(\tau)$, associated to the eigenvalue $\lambda_{k}(\tau)$, there holds $\tilde{e}_{k}^{2}(\tau, \cdot)=$ $\lambda_{k}(\tau) \tilde{e}_{k}^{1}(\tau, \cdot)$. Moreover, from the estimate $(18), \lambda_{k}(\tau) \sim i k \pi / L$ as $k$ tends to $+\infty$, uniformly for $\tau \in[0,1]$. Hence, there exists a constant $C_{1}$ so that, if $j \neq k,|j|>n_{0},|k|>n_{0}$, then

$$
\left|\alpha_{j}^{k}(\tau)\right| \leq \frac{C_{1}}{|k(j-k)|}
$$

for every $\tau \in[0,1]$.

Second case. If $|j| \leq n_{0}$, then it follows from Lemma 2 that

$$
\widetilde{A}(\tau)^{*} \tilde{f}_{j}(\tau, \cdot) \in \operatorname{Span}\left\{\tilde{f}_{p}(\tau, \cdot) \mid-n_{0} \leq p \leq n_{0}\right\},
$$

for every $\tau \in[0,1]$. Thus,

$$
\widetilde{A}(\tau)^{*} \tilde{f}_{j}(\tau, \cdot)=\sum_{p=-n_{0}}^{n_{0}} \beta_{p}^{j}(\tau) \tilde{f}_{p}(\tau, \cdot)
$$

where

$$
\beta_{p}^{j}(\tau)=\left\langle\widetilde{A}(\tau)^{*} \tilde{f}_{j}(\tau, \cdot), \tilde{e}_{p}(\tau, \cdot)\right\rangle_{H}=\left\langle\tilde{f}_{j}(\tau, \cdot), \widetilde{A}(\tau) \tilde{e}_{p}(\tau, \cdot)\right\rangle_{H} .
$$

It is not difficult to see that all coefficients $\beta_{p}^{j}(\tau)$, with $p, j \in\left\{-n_{0}, \ldots, n_{0}\right\}$, are bounded, uniformly for $\tau \in[0,1]$.

Then, (27) yields

$$
\sum_{p=-n_{0}}^{n_{0}} \beta_{p}^{j}(\tau) \alpha_{p}^{k}(\tau)=\lambda_{k}(\tau) \alpha_{j}^{k}(\tau)-\left\langle\tilde{f}_{j}(\tau, \cdot), \widetilde{A}^{\prime}(\tau) \tilde{e}_{k}(\tau, \cdot)\right\rangle_{H}
$$

Setting

$$
X(\tau):=\left(\begin{array}{c}
\alpha_{-n_{0}}^{k}(\tau) \\
\vdots \\
\alpha_{n_{0}}^{k}(\tau)
\end{array}\right), \text { and } Y(\tau):=\left(\begin{array}{c}
\left\langle\tilde{f}_{-n_{0}}(\tau, \cdot), \widetilde{A}^{\prime}(\tau) \tilde{e}_{k}(\tau, \cdot)\right\rangle_{H} \\
\vdots \\
\left\langle\tilde{f}_{n_{0}}(\tau, \cdot), \widetilde{A}^{\prime}(\tau) \tilde{e}_{k}(\tau, \cdot)\right\rangle_{H}
\end{array}\right)
$$

the latter equations can be written as

$$
\left(\lambda_{k}(\tau) I+M(\tau)\right) X(\tau)=Y(\tau),
$$

where the matrix $M(\tau)$ is bounded, uniformly for $\tau \in[0,1]$. If $|k|$ is large enough, then $\left|\lambda_{k}(\tau)\right| \sim$ $|k| \pi / L$, thus the matrix $\left(\lambda_{k}(\tau) I+M(\tau)\right)$ is invertible, and this yields readily the estimate

$$
\left|\alpha_{j}^{k}(\tau)\right| \leq \frac{C_{2}}{k^{2}},
$$

for every $\tau \in[0,1]$, and for all integers $j, k$ so that $|k|>n_{0}$ and $|j| \leq n_{0}$, where $C_{2}$ is a constant.

Finally, let us estimate $\alpha_{k}^{k}(\tau)$, for $|k|>n_{0}$. From Lemma $2,\left\|\tilde{e}_{k}(\tau, \cdot)\right\|_{H}=1$, and hence, if $|k|>n_{0}$, one gets, by differentiation with respect to $\tau$,

$$
\left\langle\tilde{e}_{k \tau}(\tau, \cdot), \tilde{e}_{k}(\tau, \cdot)\right\rangle_{H}=0
$$

From (26), we infer that

$$
\alpha_{k}^{k}(\tau)=-\sum_{\substack{j \in \mathbb{Z} \\ j \neq k}} \alpha_{j}^{k}(\tau)\left\langle\tilde{e}_{k}(\tau, \cdot), \tilde{e}_{j}(\tau, \cdot)\right\rangle_{H}
$$


Therefore, there exist constants $C_{3}$ and $C_{4}$ such that

$$
\begin{aligned}
\left|\alpha_{k}^{k}(\tau)\right| & =\left|\left\langle\tilde{e}_{k}(\tau, \cdot), \sum_{\substack{j \in \mathbb{Z} \\
j \neq k}} \alpha_{j}^{k}(\tau) \tilde{e}_{j}(\tau, \cdot)\right\rangle_{H}\right| \\
& \leq\left\|\sum_{\substack{j \in \mathbb{Z} \\
j \neq k}} \alpha_{j}^{k}(\tau) \tilde{e}_{j}(\tau, \cdot)\right\|_{H} \\
& \leq C_{3}\left(\sum_{\substack{j \in \mathbb{Z} \\
j \neq k}}\left|\alpha_{j}^{k}(\tau)\right|^{2}\right)^{1 / 2} \leq \frac{C_{4}}{|k|},
\end{aligned}
$$

for every $\tau \in[0,1]$.

It then follows from (26), and from the estimates (29), (30), and (32), that

$$
\left\|\tilde{e}_{k \tau}(\tau, \cdot)\right\|_{H}=\left\|\sum_{j \in \mathbb{Z}} \alpha_{j}^{k}(\tau) \tilde{e}_{j}(\tau, \cdot)\right\|_{H} \leq C_{3}\left(\sum_{j \in \mathbb{Z}}\left|\alpha_{j}^{k}(\tau)\right|^{2}\right)^{1 / 2} \leq \frac{C}{|k|},
$$

where $C$ is a constant.

A similar reasoning is done for $\left\|\tilde{f}_{k \tau}(\tau, \cdot)\right\|_{H}$. The lemma is proved.

Lemma 2 states the existence of a Riesz basis of $H$, consisting of generalized eigenfunctions

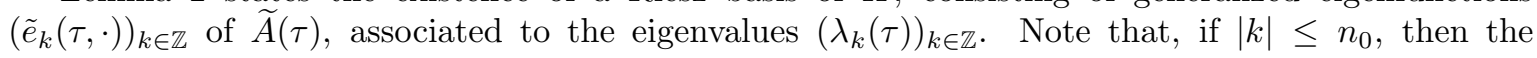
function

$$
\begin{aligned}
{[0,1] } & \rightarrow H \\
\tau & \mapsto \tilde{e}_{k}(\tau, \cdot)
\end{aligned}
$$

may fail to be of class $C^{1}$, since the corresponding eigenvalue $\lambda_{k}(\tau)$ is not necessarily algebraically simple.

However, our proof of Theorem 1 requires the existence of a Riesz basis $\left(e_{k}(\tau, \cdot)\right)_{k \in \mathbb{Z}}$, satisfying the conclusions of Lemma 2, and such that, for every integer $k$, the function $\tau \mapsto e_{k}(\tau, \cdot)$ is of class $C^{1}$.

Hence, we next modify the generalized eigenfunctions $\tilde{e}_{k}(\tau, \cdot)$, for $|k| \leq n_{0}$, so as to obtain new vectors $e_{k}(\tau, \cdot),|k| \leq n_{0}$, that are $C^{1}$ functions of $\tau$, but are not necessarily generalized eigenfunctions of $\widetilde{A}(\tau)$. The same is done for the dual Riesz basis $\left(\tilde{f}_{k}(\tau, \cdot)\right)_{k \in \mathbb{Z}}$. More precisely, we prove the following lemma.

Lemma 5. There exist a Riesz basis $\left(e_{k}(\tau, \cdot)\right)_{k \in \mathbb{Z}}$ of $H$, having a dual Riesz basis $\left(f_{k}(\tau, \cdot)\right)_{k \in \mathbb{Z}}$, such that:

(i) $e_{k}(\tau, \cdot) \in D(\widetilde{A}(\tau))$, and $\left\|e_{k}(\tau, \cdot)\right\|_{H}=1$, for every $k \in I$ and every $\tau \in[0,1]$;

(ii) for every integer $k$, the functions $\tau \mapsto e_{k}(\tau, \cdot)$ and $\tau \mapsto f_{k}(\tau, \cdot)$ are of class $C^{1}$ on $[0,1]$;

(iii) if $|k|>n_{0}$, then $e_{k}(\tau, \cdot)$ is an eigenfunction of $\widetilde{A}(\tau)$, associated to the (algebraically simple) eigenvalue $\lambda_{k}(\tau)$, and $f_{k}(\tau, \cdot)$ is an eigenfunction of $\widetilde{A}(\tau)^{*}$, associated to the (algebraically simple) eigenvalue $\overline{\lambda_{k}(\tau)}$;

(iv) for every integer $k>n_{0}$ and every $\tau \in[0,1]$, one has $e_{k}(\tau, \cdot)=\overline{e_{-k}(\tau, \cdot)}$ and $f_{k}(\tau, \cdot)=\overline{f_{-k}(\tau, \cdot)}$;

(v) for every integer $k$ so that $|k| \leq n_{0}$, there holds

$$
\widetilde{A}(\tau) e_{k}(\tau, \cdot) \in \operatorname{Span}\left\{e_{p}(\tau, \cdot)|| p \mid \leq n_{0}\right\},
$$

and

$$
\widetilde{A}(\tau)^{*} f_{k}(\tau, \cdot) \in \operatorname{Span}\left\{f_{p}(\tau, \cdot)|| p \mid \leq n_{0}\right\}
$$


Proof. For every integer $k$ so that $|k|>n_{0}$, set

$$
e_{k}(\tau, \cdot):=\tilde{e}_{k}(\tau, \cdot) \text {, and } f_{k}(\tau, \cdot):=\tilde{f}_{k}(\tau, \cdot) .
$$

Then, items (i), (ii) and (iii) hold. Moreover, for $|k|>n_{0}$, the functions $\tau \mapsto e_{k}(\tau, \cdot)$ and $\tau \mapsto f_{k}(\tau, \cdot)$ are of class $C^{1}$. We proceed with an induction argument. Assume that, for every $\tau \in[0,1]$, the subspace of $H$

$$
E(\tau):=\overline{\operatorname{Span}\left\{e_{k}(\tau, \cdot)|| k \mid>n_{0}\right\}}
$$

is of codimension $2 n_{0}+1$. Let us construct $e_{n_{0}} \in C^{1}([0,1], H)$.

We first prove that there exists $x \in C^{1}([0,1], H)$ such that $x(\tau) \notin E(\tau)$, for every $\tau \in[0,1]$. Since there does not exist necessarily an element $a \in H$ such that $a \notin E(\tau)$ for every $\tau \in[0,1]$, we deal with a subdivision of $[0,1]$, and construct $x$ using piecewise constant functions. By a compactness argument, it is clear that there exists an integer $m$, and elements $a_{1}, \ldots, a_{m}$ of $H$, such that $a_{i} \notin E(\tau)$, for every $\tau \in\left[\frac{i-1}{m}, \frac{i+1}{m}\right]$, and every $i \in\{1, \ldots, m-1\}$.

Assume that there exists $t_{1} \in[0,1]$ such that $t_{1} a_{1}+\left(1-t_{1}\right) a_{2} \in E\left(\frac{3}{2 m}\right)$. Clearly, $0<t_{1}<1$. Then, $t a_{1}-(1-t) a_{2} \notin E\left(\frac{3}{2 m}\right)$, for every $t \in[0,1]$. Indeed, by contradiction, assume that there exists $t_{2} \in[0,1]$ such that $t_{2} a_{1}-\left(1-t_{2}\right) a_{2} \in E\left(\frac{3}{2 m}\right)$. Necessarily, $0<t_{2}<1$. Then, the linear combination

$$
\frac{1-t_{2}}{1-t_{1}}\left(t_{1} a_{1}+\left(1-t_{1}\right) a_{2}\right)+t_{2} a_{1}-\left(1-t_{2}\right) a_{2}=\left(t_{1} \frac{1-t_{2}}{1-t_{1}}+t_{2}\right) a_{1}
$$

is an element of $E\left(\frac{3}{2 m}\right)$. This yields a contradiction, since $a_{1} \notin E\left(\frac{3}{2 m}\right)$.

Finally, replacing if necessary $a_{2}$ by $-a_{2}$, we proved that

$$
t a_{1}+(1-t) a_{2} \notin E\left(\frac{3}{2 m}\right),
$$

for every $t \in[0,1]$.

Then, using an easy induction argument, we may assume that

$$
t a_{i}+(1-t) a_{i+1} \notin E\left(\frac{2 i+1}{2 m}\right), i=1, \ldots, m-2,
$$

for every $t \in[0,1]$ (see Figure 1).

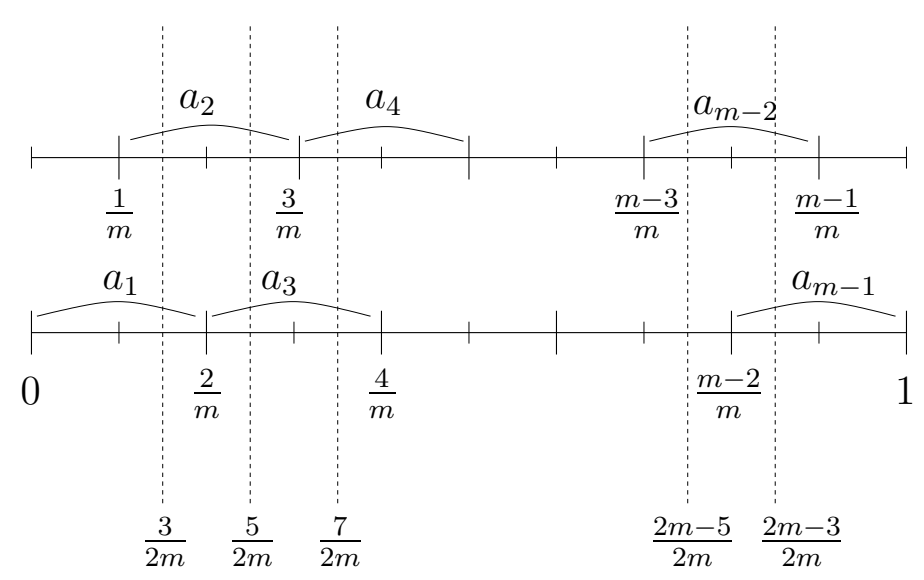

Figure 1: Construction

Therefore, by continuity, there exists $\varepsilon>0$ such that

$$
t a_{i}+(1-t) a_{i+1} \notin E(\tau),
$$


for every $\tau \in\left[\frac{2 i+1}{2 m}-\varepsilon, \frac{2 i+1}{2 m}+\varepsilon\right]$, and every $i \in\{1, \ldots, m-2\}$. As a consequence, the function $y$, defined as a piecewise linear continuous function, by

$$
y(\tau):=\left\{\begin{array}{ccc}
a_{1} & \text { if } \quad 0 \leq \tau \leq \frac{3}{2 m}-\varepsilon, \\
a_{i} & \text { if } \quad \frac{i-1}{m}+\varepsilon \leq \tau \leq \frac{i+1}{m}-\varepsilon, \\
a_{m-1} & \text { if } \quad \frac{2 m-3}{2 m}+\varepsilon \leq \tau \leq 1, \\
\frac{a_{i+1}-a_{i}}{2 \varepsilon} \tau+a_{i}-\frac{a_{i+1}-a_{i}}{2 \varepsilon}\left(\frac{2 i+1}{2 m}-\varepsilon\right) & \text { if } \quad \frac{2 i+1}{2 m}-\varepsilon \leq \tau \leq \frac{2 i+1}{2 m}+\varepsilon,
\end{array}\right.
$$

satisfies $y(\tau) \notin E(\tau)$, for every $\tau \in[0,1]$.

Using a convolution argument, we easily deduce the existence of $x \in C^{1}([0,1], H)$ such that $x(\tau) \notin E(\tau)$, for every $\tau \in[0,1]$.

Define $e_{n_{0}}:[0,1] \times[0, L] \rightarrow H$ by

$$
e_{n_{0}}(\tau, \cdot):=\frac{x(\tau)-\sum_{|k|>n_{0}}\left\langle f_{k}(\tau, \cdot), x(\tau)\right\rangle_{H} e_{k}(\tau, \cdot)}{\left\|x(\tau)-\sum_{|k|>n_{0}}\left\langle f_{k}(\tau, \cdot), x(\tau)\right\rangle_{H} e_{k}(\tau, \cdot)\right\|_{H}} .
$$

Using the estimate (25) of Lemma 4, the function $\tau \mapsto e_{n_{0}}(\tau, \cdot)$ is well defined, is of class $C^{1}$, and, by construction,

$$
\left\|e_{n_{0}}(\tau, \cdot)\right\|_{H}=1
$$

and

$$
\left\langle f_{k}(\tau, \cdot), e_{n_{0}}(\tau, \cdot)\right\rangle_{H}=0
$$

for $|k|>n_{0}$, and for every $\tau \in[0,1]$.

Define $f_{n_{0}}:[0,1] \times[0, L] \rightarrow H$ by

$$
f_{n_{0}}(\tau, \cdot):=e_{n_{0}}(\tau, \cdot)-\sum_{|k|>n_{0}}\left\langle e_{n_{0}}(\tau, \cdot), e_{k}(\tau, \cdot)\right\rangle_{H} f_{k}(\tau, \cdot) .
$$

Using the estimate (25) of Lemma 4, the function $\tau \mapsto f_{n_{0}}(\tau, \cdot)$ is well defined, is of class $C^{1}$, and one has

$$
\left\langle f_{n_{0}}(\tau, \cdot), e_{n_{0}}(\tau, \cdot)\right\rangle_{H}=1
$$

and

$$
\left\langle f_{n_{0}}(\tau, \cdot), e_{k}(\tau, \cdot)\right\rangle_{H}=0
$$

for $|k|>n_{0}$, and for every $\tau \in[0,1]$.

For all integers $k, l$ so that $|k| \leq n_{0}$ and $|l|>n_{0}$, there holds, by construction,

$$
\left\langle f_{l}(\tau, \cdot), \widetilde{A}(\tau) e_{k}(\tau, \cdot)\right\rangle_{H}=\left\langle\widetilde{A}(\tau)^{*} f_{l}(\tau, \cdot), e_{k}(\tau, \cdot)\right\rangle_{H}=\lambda_{l}(\tau)\left\langle f_{l}(\tau, \cdot), e_{k}(\tau, \cdot)\right\rangle_{H}=0,
$$

and the item (v) follows easily.

Remark 11. Denote $e_{k}(\tau, \cdot)=\left(\begin{array}{c}e_{k}^{1}(\tau, \cdot) \\ e_{k}^{2}(\tau, \cdot)\end{array}\right)$, for every integer $k$. If $|k|>n_{0}$, then, from (iii) in Lemma 5 , there holds

$$
e_{k}^{2}(\tau, \cdot)=\lambda_{k}(\tau) e_{k}^{1}(\tau, \cdot),
$$


and

$$
\begin{aligned}
& e_{k x x}^{1}(\tau, \cdot)+f^{\prime}(\hat{y}(\tau, \cdot)) e_{k}^{1}(\tau, \cdot)=\lambda_{k}(\tau)^{2} e_{k}^{1}(\tau, \cdot) \\
& e_{k}^{1}(\tau, 0)=0, e_{k x}^{1}(\tau, L)=-\alpha \lambda_{k}(\tau) e_{k}^{1}(\tau, L)
\end{aligned}
$$

Similarly, denote $f_{j}(\tau, \cdot)=\left(\begin{array}{c}f_{j}^{1}(\tau, \cdot) \\ \left.f_{j}^{2}(\tau, \cdot)\right)\end{array}\right)$, for every integer $j$. If $|j|>n_{0}$, then

$$
\left\{\begin{array}{l}
f_{j_{x x}}^{2}(\tau, \cdot)+f^{\prime}(\hat{y}(\tau, \cdot)) f_{j}^{2}(\tau, \cdot)={\overline{\lambda_{j}(\tau)}}^{2} f_{j}^{2}(\tau, \cdot), \\
f_{j}^{2}(\tau, 0)=0, f_{j_{x}}^{2}(\tau, L)=-\alpha \overline{\lambda_{j}(\tau)} f_{j}^{2}(\tau, L),
\end{array}\right.
$$

and

$$
\left\{\begin{array}{l}
f_{j_{x x}}^{1}(\tau, \cdot)=-\overline{\lambda_{j}(\tau)} f_{j}^{2}(\tau, \cdot), \\
f_{j}^{1}(\tau, 0)=0, f_{j_{x}}^{1}(\tau, L)=\alpha f_{j}^{2}(\tau, L),
\end{array}\right.
$$

for every $\tau \in[0,1]$.

\subsection{The finite dimensional unstable part of the system}

Let $\alpha>1$ so that

$$
\frac{1}{2 L} \ln \frac{\alpha-1}{\alpha+1}<-1
$$

Using (18), only a finite number of eigenvalues may have a nonnegative real part as $\tau \in[0,1]$. More precisely, there exists an integer $n$ so that

$$
\forall \tau \in[0,1], \quad \forall k \in \mathbb{Z}, \quad(|k|>n) \Rightarrow\left(\operatorname{Re}\left(\lambda_{k}(\tau)\right)<-1\right) .
$$

Without loss of generality, we suppose that $n \geq n_{0}$. Therefore, from Lemma 5 , each eigenvalue $\lambda_{k}(\tau)$, with $|k|>n$, is algebraically simple, and satisfies $\operatorname{Re}\left(\lambda_{k}(\tau)\right)<-1$.

Remark 12. Note that the integer $n$ can be arbitrarily large. For example if $f(y)=y^{3}$ and if $y_{1}^{\prime}(0) \rightarrow+\infty$ then $n \rightarrow+\infty$.

Every solution $W(t, \cdot) \in D(\widetilde{A}(\tau))$ of $(15)$ can be expanded as series in the Riesz basis $\left(e_{j}(\varepsilon t, \cdot)\right)_{j \in \mathbb{Z}}$ of $H$, convergent in $H$,

$$
W(t, \cdot)=\left(\begin{array}{l}
w^{1}(t, \cdot) \\
w^{2}(t, \cdot)
\end{array}\right)=\sum_{j=-\infty}^{\infty} w_{j}(t) e_{j}(\varepsilon t, \cdot)
$$

Note that, for integers $k$ satisfying $|k| \leq n$, the eigenvalue $\lambda_{k}(\tau)$ may be real, and/or non algebraically simple. Since $W(t, x) \in \mathbb{R}^{2}$, one has $w_{j}(t)=\overline{w_{-j}(t)}$, for every $j>n$, and hence

$$
W(t, \cdot)=\pi_{1}(\varepsilon t) W(t, \cdot)+2 \operatorname{Re}\left(\sum_{j=n+1}^{+\infty} w_{j}(t) e_{j}(\varepsilon t, \cdot)\right),
$$

where $\pi_{1}(\tau)$ denotes the projection from $H$ onto $\operatorname{Span}\left\{e_{p}(\tau, \cdot)|| p \mid \leq n\right\}$, defined by

$$
\pi_{1}(\tau) h=\sum_{j=-n}^{n}\left\langle f_{j}(\tau, \cdot), h\right\rangle e_{j}(\tau, \cdot)
$$

for every $h \in H$. By construction, it is quite clear that $\widetilde{A}(\tau)$ and $\pi_{1}(\tau)$ commute. In what follows, $\operatorname{Im} \pi_{1}(\tau)$ is identified to $\mathbb{R}^{2 \mathrm{n}+1}$, and we denote by $\widetilde{A}_{1}(\tau)$ the $(2 n+1) \times(2 n+1)$ matrix (with real coefficients) representing the restriction of $\widetilde{A}(\tau)$ on $\operatorname{Im} \pi_{1}(\tau)$. 
Lemma 6. The mapping

$$
\begin{aligned}
{[0,1] } & \rightarrow L(H, H) \\
\tau & \mapsto \pi_{1}(\tau)
\end{aligned}
$$

is of class $C^{1}$, and one has

$$
\pi_{1}^{\prime}(\tau) h=-\sum_{|j|>n}\left\langle f_{j_{\tau}}(\tau, \cdot), h\right\rangle_{H} e_{j}(\tau, \cdot)-\sum_{|j|>n}\left\langle f_{j}(\tau, \cdot), h\right\rangle_{H} e_{j_{\tau}}(\tau, \cdot),
$$

for every $h \in H$.

Proof. For every $h \in H$, and every $\tau \in[0,1]$, one has, using Lemma 5 ,

$$
\pi_{1}(\tau) h=h-\sum_{|j|>n}\left\langle f_{j}(\tau, \cdot), h\right\rangle_{H} e_{j}(\tau, \cdot) .
$$

For $|j|>n \geq n_{0}$, the eigenfunctions $e_{j}(\tau, \cdot)$ and $f_{j}(\tau, \cdot)$ are $C^{1}$ functions of $\tau$. Using the estimates 25 of Lemma 4, the sum

$$
-\sum_{|j|>n}\left\langle f_{j_{\tau}}(\tau, \cdot), h\right\rangle_{H} e_{j}(\tau, \cdot)-\sum_{|j|>n}\left\langle f_{j}(\tau, \cdot), h\right\rangle_{H} e_{j_{\tau}}(\tau, \cdot)
$$

converges normally, and the conclusion follows.

In the sequel, we are going to move, by means of an appropriate feedback control, the $2 n+1$ eigenvalues $\lambda_{0}(\tau), \ldots, \lambda_{n}(\tau)$, whose real part may be nonnegative, without moving the others, so that all eigenvalues then have a negative real part. This pole-shifting process is the first part of the stabilization procedure (see for instance $[12,15]$ for details on this standard theory).

Set $W_{1}(t)=\pi_{1}(\varepsilon t) W(t, \cdot)$. Then, from $(15)$,

$$
W_{1}^{\prime}(t)=\widetilde{A}_{1}(\varepsilon t) W_{1}(t)+v(t) a_{1}(\varepsilon t)+v^{\prime \prime}(t) b_{1}(\varepsilon t)+r_{1}(\varepsilon, t)
$$

where

$$
a_{1}(\varepsilon t)=\pi_{1}(\tau) a(\varepsilon t, \cdot), b_{1}(\varepsilon t)=\pi_{1}(\varepsilon t) b(\cdot), r_{1}(\varepsilon, t)=\pi_{1}(\varepsilon t) R(\varepsilon, t, \cdot)+\varepsilon \pi^{\prime}(\varepsilon t) W(t, \cdot) .
$$

Lemma 7. There exists a constant $C_{3}$ such that, if $|v(t)|+\|w(t, \cdot)\|_{L^{\infty}(0, L)} \leq 1$, then

$$
\left\|r_{1}(\varepsilon, t)\right\|_{H} \leq C_{3}\left(\varepsilon^{2}+v(t)^{2}+\|W(t, \cdot)\|_{H}^{2}\right)
$$

for every $t \in[0,1 / \varepsilon]$.

Proof. The estimate follows from Lemma 6 , from the definition $(16)$ of $R(\varepsilon, t, \cdot)$, and from the estimate (9).

The system (38) is a differential system in $\mathbb{R}^{2 \mathrm{n}+1}$ controlled by $v, v^{\prime}, v^{\prime \prime}$. Set

$$
\beta(t):=v^{\prime}(t), \gamma(t):=v^{\prime \prime}(t)
$$

and consider now $v(t)$ and $\beta(t)$ as state coordinates, and $\gamma(t)$ as a control. Notice that $v(t), \beta(t)$ and $\gamma(t)$ are real numbers. Then, the former finite dimensional system may be rewritten as

$$
\left\{\begin{aligned}
v^{\prime}(t) & =\beta(t) \\
\beta^{\prime}(t) & =\gamma(t) \\
W_{1}^{\prime}(t) & =\widetilde{A}_{1}(\varepsilon t) W_{1}(t)+a_{1}(\varepsilon t) v(t)+b_{1}(\varepsilon t) \gamma(t)+r_{1}(\varepsilon, t) .
\end{aligned}\right.
$$


Introducing the matrix notations

$$
X_{1}(t)=\left(\begin{array}{c}
v(t) \\
\beta(t) \\
W_{1}(t)
\end{array}\right), A_{1}(\tau)=\left(\begin{array}{ccc}
0 & 1 & 0 \\
0 & 0 & 0 \\
a_{1}(\tau) & 0 & \widetilde{A}_{1}(\tau)
\end{array}\right), B_{1}(\tau)=\left(\begin{array}{c}
0 \\
1 \\
b_{1}(\tau)
\end{array}\right), R_{1}(\varepsilon, t)=\left(\begin{array}{c}
0 \\
0 \\
r_{1}(\varepsilon, t)
\end{array}\right),
$$

we obtain

$$
X_{1}^{\prime}(t)=A_{1}(\varepsilon t) X_{1}(t)+B_{1}(\varepsilon t) \gamma(t)+R_{1}(\varepsilon, t) .
$$

Lemma 8. For each $\tau \in[0,1]$, the pair $\left(A_{1}(\tau), B_{1}(\tau)\right)$ satisfies the Kalman condition, i.e.

$$
\operatorname{det}\left(B_{1}(\tau), A_{1}(\tau) B_{1}(\tau), \ldots, A_{1}(\tau)^{2 n+2} B_{1}(\tau)\right) \neq 0 .
$$

Proof of Lemma 8. Let $\tau \in[0,1]$ be fixed. Consider the infinite dimensional linear control system

$$
\left\{\begin{aligned}
v^{\prime}(t) & =\beta(t) \\
\beta^{\prime}(t) & =\gamma(t) \\
w_{t}(t, x) & =\widetilde{A}(\tau) w(t, x)+v(t) a(\tau, x)+\gamma(t) b(x)
\end{aligned}\right.
$$

where the state is $(v(t), \beta(t), w(t, \cdot)) \in \mathbb{R} \times \mathbb{R} \times \mathrm{H}$, and the control is $\gamma(t) \in \mathbb{R}$. It is clear from Section 2.2 that this control system is equivalent to the boundary control system

$$
\left\{\begin{array}{l}
z_{t t}(t, x)=z_{x x}(t, x)+f^{\prime}(\bar{y}(\tau, x)) z(t, x) \\
z(t, 0)=0, z_{x}(t, L)=-\alpha z_{t}(t, L)+v(t) \\
v^{\prime}(t)=\beta(t), \beta^{\prime}(t)=\gamma(t)
\end{array}\right.
$$

which is a classical linear wave equation. Let $T>2 L$. It is well known that the linear control system (46), and hence the linear control system (45), is exactly controllable in time $T$ (see [2]), namely, for all $\left(v_{0}, \beta_{0}, w_{0}\right)$ and $\left(v_{1}, \beta_{1}, w_{1}\right)$ in $\mathbb{R} \times \mathbb{R} \times \mathrm{H}$, there exists a control $\gamma \in L^{2}(0, T)$ such that the solution $(v, \beta, w)$ of $(45)$ associated to this control, starting from $(v(0), \beta(0), w(0, \cdot))=\left(v_{0}, \beta_{0}, w_{0}\right)$, satisfies $(v(T), \beta(T), w(T, \cdot))=\left(v_{1}, \beta_{1}, w_{1}\right)$. This implies in particular that the finite dimension linear control system

$$
\dot{X}_{1}(t)=A_{1}(\tau) X_{1}(t)+B_{1}(\tau) \gamma(t)
$$

is controllable in time $T$. Hence, the Kalman condition (44) holds.

It is well known that, for an autonomous finite dimensional linear control system, the Kalman condition, equivalent to the controllability of the system, implies the stabilizability of the system. This is not longer true for nonautonomous linear systems; however, this holds provided that the system is slowly time varying, whence the importance of the parameter $\varepsilon$. In these conditions, Lemma 8 implies the following result (see [12, Chap. 9.6]).

Corollary 2. There exists a $C^{1}$ mapping $\tau \mapsto K_{1}(\tau)$ on $[0,1]$, where $K_{1}(\tau)$ is a $1 \times(2 n+1)$ matrix with real coefficients, such that the matrix $A_{1}(\tau)+B_{1}(\tau) K_{1}(\tau)$ admits -1 as an eigenvalue of order $2 n+3$, for every $\tau \in[0,1]$.

Moreover, there exists a $C^{1}$ mapping $\tau \mapsto P(\tau)$ on $[0,1]$, where $P(\tau)$ is a $(2 n+3) \times(2 n+3)$ symmetric positive definite real matrix, such that the identity

$$
P(\tau)\left(A_{1}(\tau)+B_{1}(\tau) K_{1}(\tau)\right)+{ }^{t}\left(A_{1}(\tau)+B_{1}(\tau) K_{1}(\tau)\right) P(\tau)=-I
$$

holds, for every $\tau \in[0,1]$.

The gain matrix $K_{1}(\tau)$ permits to construct on $[0,1 / \varepsilon]$ the feedback control function

$$
\gamma(t)=K_{1}(\varepsilon t) X_{1}(t)
$$

that stabilizes the finite dimensional control system (43). We next prove that this feedback actually stabilizes the whole infinite dimensional system (15), provided $\varepsilon>0$ is small enough. 


\subsection{Construction of a Lyapunov functional}

Let us first write the differential equation satisfied by each (complex) coordinate $w_{j}(t)=\left\langle f_{j}(\varepsilon t, \cdot), W(t, \cdot)\right\rangle_{H}$, with $j>n$. There holds

$$
w_{j}^{\prime}(t)=\left\langle f_{j}(\varepsilon t, \cdot), W_{t}(t, \cdot)\right\rangle_{H}+\varepsilon\left\langle f_{j_{\tau}}(\varepsilon t, \cdot), W(t, \cdot)\right\rangle_{H} .
$$

Since

$$
\widetilde{A}(\varepsilon t) W(t, \cdot)=\pi_{1}(\varepsilon t) W(t, \cdot)+\sum_{|j|>n} \lambda_{j}(\varepsilon t) w_{j}(t) e_{j}(\varepsilon t, \cdot)
$$

we get, using (15) and (16),

$$
\left\langle f_{j}(\varepsilon t, \cdot), W_{t}(t, \cdot)\right\rangle_{H}=\lambda_{j}(\varepsilon t) w_{j}(t)+a_{j}(\varepsilon t) v(t)+b_{j}(\varepsilon t) v^{\prime \prime}(t)+\left\langle f_{j}(\varepsilon t, \cdot), R(\varepsilon, t, \cdot)\right\rangle_{H},
$$

where

$$
\begin{aligned}
& a_{j}(\varepsilon t):=\left\langle f_{j}(\varepsilon t, \cdot), a(\varepsilon t, \cdot)\right\rangle_{H}=\int_{0}^{L} \overline{f_{j}^{2}(\varepsilon t, x)}\left(\frac{x(x-L)}{L} f^{\prime}(\hat{y}(\varepsilon t, x))+\frac{2}{L}\right) d x, \\
& b_{j}(\varepsilon t):=\left\langle f_{j}(\varepsilon t, \cdot), b(\cdot)\right\rangle_{H}=-\int_{0}^{L} \overline{f_{j}^{2}(\varepsilon t, x)} \frac{x(x-L)}{L} d x .
\end{aligned}
$$

Equation (49) thus yields, for every $j>n$,

$$
w_{j}^{\prime}(t)=\lambda_{j}(\varepsilon t) w_{j}(t)+a_{j}(\varepsilon t) v(t)+b_{j}(\varepsilon t) v^{\prime \prime}(t)+r_{j}(\varepsilon, t),
$$

where

$$
r_{j}(\varepsilon, t):=\left\langle f_{j}(\varepsilon t, \cdot), R(\varepsilon, t, \cdot)\right\rangle_{H}+\varepsilon\left\langle f_{j_{\tau}}(\varepsilon t, \cdot), W(t, \cdot)\right\rangle_{H} .
$$

The aim is now to construct a control Lyapunov functional in order to stabilize system (15), using the feedback control (48). For every $t \in[0,1 / \varepsilon]$, all $v, \beta \in \mathbb{R}$, and every $W(\cdot)=\left(\begin{array}{l}w^{1}(\cdot) \\ w^{2}(\cdot)\end{array}\right) \in H$, we set

$$
E(t, v, \beta, W(\cdot)):={ }^{t} X_{1}(t) P(\varepsilon t) X_{1}(t)
$$

where $X_{1}(t)$ denotes the matrix vector in $\mathbb{C}^{2 n+3}$

$$
X_{1}(t):=\left(\begin{array}{c}
v \\
\beta \\
\pi_{1}(\varepsilon t) W(\cdot)
\end{array}\right) .
$$

For every $j \in \mathbb{Z}$, set

$$
w_{j}(t):=\left\langle f_{j}(\varepsilon t, \cdot), W(\cdot)\right\rangle_{H},
$$

and define

$$
N(t, W(\cdot)):=\frac{1}{2} \sum_{|j|>n}\left|w_{j}(t)\right|^{2},
$$

where $|\cdot|$ denotes the complex modulus. Finally, introduce

$$
V(t, v, \beta, W(\cdot)):=c E(t, v, \beta, W(\cdot))+N(t, W(\cdot))
$$

where $c$ is a positive real number to be fixed later.

The rest of the section is devoted to prove that $V$ is a Lyapunov functional for the system (15), with the feedback control (48).

In what follows we will repeatedly use the equivalence of norms in finite dimension. The following notation will thus happen to be useful. 
Notation. Let $\Lambda$ be a set and $\Delta=\{(\varepsilon, t) \mid 0<\varepsilon \leq 1,0 \leq t \leq 1 / \varepsilon\}$. Let $F_{1}, F_{2}$ and $F_{3}$ be real functions defined on $\Delta \times \Lambda$, and let $\theta \in[0,+\infty]$. The notation $F_{1} \lesssim F_{2}$ on $F_{3} \leq \theta$ means that $F_{2} \geq 0$ and that there exists a positive constant $C$ such that

$$
\forall(\varepsilon, t) \in \Delta \quad \forall \lambda \in \Lambda \quad\left(F_{3}(\varepsilon, t, \lambda) \leq \theta\right) \Rightarrow\left(\left|F_{1}(\varepsilon, t, \lambda)\right| \leq C F_{2}(\varepsilon, t, \lambda)\right) .
$$

We say that $F_{1} \sim F_{2}$ if both $F_{1} \lesssim F_{2}$ and $F_{2} \lesssim F_{1}$ hold on $F_{3} \leq \theta$.

For the sake of simplicity, when the set $\Lambda$ is clear from the context it will not be given explicitly.

Let $\|\cdot\|_{2}$ denote the Hermitian norm in $\mathbb{C}^{2 n+3}$. Since $P(\tau)$ is real symmetric positive definite, we can write (with $\Lambda=\mathbb{C}^{2 n+3}$ )

$$
E(t, v, \beta, W(\cdot))={ }^{t} \overline{X_{1}(t)} P(\varepsilon t) X_{1}(t) \sim\left\|X_{1}(t)\right\|_{2}^{2} .
$$

Since $W(\cdot)=\sum_{j \in \mathbb{Z}} w_{j}(t) e_{j}(\varepsilon t, \cdot)$, by definition of a Riesz basis (see (17)), and using the uniform property (20), we have

$$
\begin{aligned}
V(t, v, \beta, W(\cdot)) & \sim v^{2}+\beta^{2}+\sum_{j \in \mathbb{Z}}\left|w_{j}(t)\right|^{2} \\
& \sim v^{2}+\beta^{2}+\|W(t, \cdot)\|_{H}^{2} \\
& \sim v^{2}+\beta^{2}+\left\|w_{x}^{1}(\cdot)\right\|_{L^{2}(0, L)}^{2}+\left\|w^{2}(\cdot)\right\|_{L^{2}(0, L)}^{2} .
\end{aligned}
$$

Remark 13. The meaning of $V$ is the following. Except the first eigenmodes, the term $N$ is equivalent to the classical energy of the wave equation, as explained in the introduction. As was shown previously, there exists a finite number of unstable modes. The term $E$ is used to stabilize this unstable finite dimensional part of the system, and appears as a term of correction.

Let now $(v(t), \beta(t), W(t, \cdot))$ denote a solution of (15), in which we choose the control $\gamma(t)$ in the feedback form (48). Then,

$$
W_{t}(t, \cdot)=\widetilde{A}(\varepsilon t) W(t, \cdot)+a(\varepsilon t, \cdot) v(t)+b(\cdot) K_{1}(\varepsilon t) X_{1}(t)+R(\varepsilon, t, \cdot) .
$$

Set

$$
\begin{aligned}
E_{1}(t) & :=E(t, v(t), \beta(t), W(t, \cdot)), \\
N_{1}(t) & :=N(t, w(t, \cdot)), \\
V_{1}(t) & :=V(t, W(t, \cdot))=c E_{1}(t)+N_{1}(t) .
\end{aligned}
$$

Let us compute $V_{1}^{\prime}(t)$ and state a differential inequality satisfied by $V_{1}$. First of all, from (43) and (47), we get

$$
\begin{aligned}
& E_{1}^{\prime}(t)={ }^{t} \overline{X_{1}^{\prime}(t)} P(\varepsilon t) X_{1}(t)+{ }^{t} \overline{X_{1}(t)} P(\varepsilon t) X_{1}^{\prime}(t)+\varepsilon^{t} \overline{X_{1}(t)} P^{\prime}(\varepsilon t) X_{1}(t) \\
& =-\left\|X_{1}(t)\right\|_{2}^{2}+{ }^{t} \overline{R_{1}(\varepsilon, t)} P(\varepsilon t) X_{1}(t)+{ }^{t} X_{1}(t) P(\varepsilon t) R_{1}(\varepsilon, t)+\varepsilon^{t} \bar{X}_{1}(t) P^{\prime}(\varepsilon t) X_{1}(t)
\end{aligned}
$$

Using the a priori estimate (40), we infer that, if

$$
|v(t)|+\left\|w^{1}(t, \cdot)\right\|_{L^{\infty}(0, L)} \leq 1,
$$

then

$$
\left\|R_{1}(\varepsilon, t)\right\|_{2} \lesssim \varepsilon^{2}+v(t)^{2}+N_{1}(t) .
$$

Hence, if (59) holds, then

$$
\begin{aligned}
& \left|{ }^{t} R_{1}(\varepsilon, t) P(\varepsilon t) X_{1}(t)+{ }^{t} X_{1}(t) P(\varepsilon t) R_{1}(\varepsilon, t)\right| \lesssim\left\|X_{1}(t)\right\|_{2}\left(\varepsilon^{2}+v(t)^{2}+N_{1}(t)\right) \\
& \lesssim \sqrt{E_{1}(t)}\left(\varepsilon^{2}+E_{1}(t)+N_{1}(t)\right) \text {. }
\end{aligned}
$$


On the other part, from Corollary 2, the mapping $\tau \mapsto P^{\prime}(\tau)$ is bounded on $[0,1]$, hence

$$
\left|\varepsilon^{t} X_{1}(t) P^{\prime}(\varepsilon t) X_{1}(t)\right| \lesssim \varepsilon\left\|X_{1}(t)\right\|_{2}^{2} \lesssim \varepsilon E_{1}(t) \lesssim \varepsilon^{2}+E_{1}(t)^{2}
$$

Therefore, using (58), there exists $\delta_{1}>0$ such that, if (59) holds, then

$$
E_{1}^{\prime}(t)+\delta_{1} E_{1}(t) \lesssim \varepsilon^{2}+E_{1}(t)^{2}+\sqrt{E_{1}(t)}\left(\varepsilon^{2}+E_{1}(t)+N_{1}(t)\right) .
$$

Hence, there exists $\rho_{1}>0$ such that, for every $\varepsilon \in(0,1]$, and for every $t \in[0,1 / \varepsilon]$ so that $E_{1}(t)+$ $N_{1}(t) \leq \rho_{1}$,

$$
E_{1}^{\prime}(t)+\frac{\delta_{1}}{2} E_{1}(t) \lesssim \varepsilon^{2}+N_{1}(t)^{2}
$$

Let us now handle $N_{1}(t)$. From (51), we have

$$
\begin{aligned}
N_{1}^{\prime}(t) & =\operatorname{Re} \sum_{|j|>n} \overline{w_{j}(t)} w_{j}^{\prime}(t) \\
& =\sum_{|j|>n} \operatorname{Re}\left(\lambda_{j}(\varepsilon t)\right)\left|w_{j}(t)\right|^{2}+\operatorname{Re} \sum_{|j|>n} \overline{w_{j}(t)}\left(a_{j}(\varepsilon t) v(t)+b_{j}(\varepsilon t) K_{1}(\varepsilon t) X_{1}(\varepsilon t)+r_{j}(\varepsilon, t)\right) .
\end{aligned}
$$

Clearly,

$$
\begin{aligned}
\left|\sum_{|j|>n} \overline{w_{j}(t)}\left(a_{j}(\varepsilon t) v(t)+b_{j}(\varepsilon t) K_{1}(\varepsilon t) X_{1}(\varepsilon t)\right)\right| & \lesssim \sqrt{N_{1}(t)}\left(|v(t)|\|a(\varepsilon t, \cdot)\|_{H}+\left\|X_{1}(t)\right\|_{2}\|b(\cdot)\|_{H}\right) \\
& \lesssim \sqrt{N_{1}(t)} \sqrt{E_{1}(t)} .
\end{aligned}
$$

The term $\sum \overline{w_{j}} r_{j}$ is more difficult to handle. First, from (52), we have

$$
\sum_{|j|>n} \overline{w_{j}(t)} r_{j}(\varepsilon, t)=\sum_{|j|>n} \overline{w_{j}(t)}\left\langle f_{j}(\varepsilon t, \cdot), R(\varepsilon, t, \cdot)\right\rangle_{H}+\varepsilon \sum_{|j|>n} \overline{w_{j}(t)}\left\langle f_{j_{\tau}}(\varepsilon t, \cdot), W(t, \cdot)\right\rangle_{H} .
$$

Since $\left(f_{j}(\varepsilon t, \cdot)\right)_{j \in \mathbb{Z}}$ is a Riesz basis of $H$, the first term is easily estimated by

$$
\left|\sum_{|j|>n} \overline{w_{j}(t)}\left\langle f_{j}(\varepsilon t, \cdot), R(\varepsilon, t, \cdot)\right\rangle_{H}\right| \lesssim \sqrt{N_{1}(t)}\|R(\varepsilon, t, \cdot)\|_{H}
$$

and using the a priori estimate (9), and (16), we infer that

$$
\left|\sum_{|j|>n} \overline{w_{j}(t)}\left\langle f_{j}(\varepsilon t, \cdot), R(\varepsilon, t, \cdot)\right\rangle_{H}\right| \lesssim \sqrt{N_{1}(t)}\left(\varepsilon^{2}+v(t)^{2}+\|W(t, \cdot)\|_{H}^{2}\right),
$$

provided $|v(t)|+\left\|w^{1}(t, \cdot)\right\|_{L^{\infty}(0, L)} \leq 1$. Concerning the second term, we get from Lemma 4 the estimate

$$
\left|\sum_{|j|>n} \overline{w_{j}(t)}\left\langle f_{j_{\tau}}(\varepsilon t, \cdot), W(t, \cdot)\right\rangle_{H}\right| \lesssim \sqrt{N_{1}(t)}\|W(t, \cdot)\|_{H}
$$

It follows from (64), (65), and (66), that

$$
\left|\sum_{|j|>n} \overline{w_{j}(t)} r_{j}(\varepsilon, t)\right| \lesssim \sqrt{N_{1}(t)}\left(\varepsilon^{2}+v(t)^{2}+\|W(t, \cdot)\|_{H}^{2}\right)+\varepsilon \sqrt{N_{1}(t)}\|W(t, \cdot)\|_{H} .
$$

From (62), (63) and (67), we get, if (59) holds,

$$
\begin{aligned}
N_{1}^{\prime}(t)-\sum_{|j|>n} \operatorname{Re}\left(\lambda_{j}(\varepsilon t)\right)\left|w_{j}(t)\right|^{2} \lesssim & \sqrt{N_{1}(t)} \sqrt{E_{1}(t)}+\varepsilon \sqrt{N_{1}(t)}\|W(t, \cdot)\|_{H} \\
& +\sqrt{N_{1}(t)}\left(\varepsilon^{2}+v(t)^{2}+\|W(t, \cdot)\|_{H}^{2}\right) .
\end{aligned}
$$


Using the estimates

$$
\begin{aligned}
& v(t)^{2}+\|W(t, \cdot)\|_{H}^{2} \lesssim E_{1}(t)+N_{1}(t), \\
& W(t, \cdot) \|_{H} \lesssim \sqrt{E_{1}(t)}+\sqrt{N_{1}(t)},
\end{aligned}
$$

and the estimate (35) on the eigenvalues, namely, $\operatorname{Re}\left(\lambda_{j}(\varepsilon t)\right) \leq-1$ for $|j|>n$, we get from (68),

$$
\begin{gathered}
N_{1}^{\prime}(t)+N_{1}(t) \lesssim \sqrt{E_{1}(t)} \sqrt{N_{1}(t)}+\sqrt{N_{1}(t)}\left(\varepsilon^{2}+E_{1}(t)+N_{1}(t)\right) \\
+\varepsilon \sqrt{N_{1}(t)}\left(\sqrt{E_{1}(t)}+\sqrt{N_{1}(t)}\right) .
\end{gathered}
$$

Note that, for every $\theta \in(0,+\infty)$,

$$
\begin{aligned}
& \sqrt{E_{1}(t)} \sqrt{N_{1}(t)} \leq \frac{\theta}{2} N_{1}(t)+\frac{1}{2 \theta} E_{1}(t), \\
& \varepsilon^{2} \sqrt{N_{1}(t)} \leq \frac{\theta}{2} N_{1}(t)+\frac{1}{2 \theta} \varepsilon^{4}, \\
& \sqrt{N_{1}(t)} E_{1}(t) \leq \frac{\theta}{2} N_{1}(t)+\frac{1}{2 \theta} E_{1}(t)^{2} .
\end{aligned}
$$

Hence, taking $\theta>0$ small enough, using (69), we can assert the existence of positive real numbers $\varepsilon_{0}>0$ and $\rho_{2}>0$ such that, for every $\varepsilon \in\left(0, \varepsilon_{0}\right)$ and for every $t \in[0,1 / \varepsilon]$ so that $E_{1}(t)+N_{1}(t) \leq \rho_{2}$,

$$
N_{1}^{\prime}(t)+\frac{1}{2} N_{1}(t) \lesssim E_{1}(t)+\varepsilon^{2} .
$$

Using (61), and setting $\rho=\min \left(\rho_{1}, \rho_{2}\right)$, there exists $\sigma_{1}>0$ such that, for every $\varepsilon \in\left(0, \varepsilon_{0}\right)$ and for every $t \in[0,1 / \varepsilon]$ so that $E_{1}(t)+N_{1}(t) \leq \rho$, there holds, for every $c>0$,

$$
c E_{1}^{\prime}(t)+N_{1}^{\prime}(t)+\frac{\delta_{1} c}{2} E_{1}(t)+\frac{1}{2} N_{1}(t) \leq \sigma_{1}\left((1+c) \varepsilon^{2}+E_{1}(t)+c N_{1}(t)^{2}\right),
$$

Define the constant $c$ by

$$
c:=\frac{2 \sigma_{1}}{\delta_{1}} .
$$

Then, the function $V_{1}(t)=c E_{1}(t)+N_{1}(t)$ satisfies the following estimate: there exists $\rho^{\prime}>0$ such that, for every $\varepsilon \in\left(0, \varepsilon_{0}\right)$, and for every $t \in[0,1 / \varepsilon]$ so that $V_{1}(t) \leq \rho^{\prime}$, there holds

$$
V_{1}^{\prime}(t) \leq \sigma_{1}(1+c) \varepsilon^{2} .
$$

Since $v(0)=0$ and $\beta(0)=0$, one has $V_{1}(0) \lesssim \varepsilon^{2}$ (see (12)), and thus there exist $\varepsilon_{1}>0$ and $\sigma_{2}>0$ such that, for every $\varepsilon \in\left(0, \varepsilon_{1}\right]$, and for every $t \in[0,1 / \varepsilon]$,

$$
V_{1}(t) \leq \sigma_{2} \varepsilon
$$

In particular (see (4) and (6)),

$$
\left\|y(1 / \varepsilon, \cdot)-y_{1}(\cdot)\right\|_{H^{1}(0, L)}+\left\|y_{t}(1 / \varepsilon, \cdot)\right\|_{L^{2}(0, L)} \leq \gamma \varepsilon,
$$

where $\gamma>0$ is a real number not depending on $\varepsilon \in\left(0, \varepsilon_{1}\right]$. This ends the proof of Theorem 1 .

\subsection{Proof of Corollary 1}

The proof consists in solving a local exact controllability result. From the previous section, $y(1 / \varepsilon, \cdot)$ belongs to an arbitrarily small neighborhood of $y_{1}(\cdot)$ in $H^{1}$-topology if $\varepsilon$ is small enough, and our 
aim is now to construct a trajectory $q(t, x)$ solution of the control system steering $y(1 / \varepsilon, \cdot)$ to $y_{1}(\cdot)$ in some time $T>0$ (for instance $T=1$ ), i.e.

$$
\left\{\begin{array}{l}
q_{t t}=q_{x x}+f(q) \\
q(t, 0)=0, q_{x}(t, L)=u(t) \\
q(0, x)=y(1 / \varepsilon, x), q(T, x)=y_{1}(x) .
\end{array}\right.
$$

Existence of such a solution $q$ is given by [27]. Actually in [27] the function $f$ is assumed to be globally Lipschitzian, but the local result we need here readily follows from the proofs and the estimates contained in this paper.

Indeed, let $T>0$ and let $\tilde{f}$ be a globally Lipschitzian mapping such that

$$
\tilde{f}(s)=f(s), \quad \forall s \in\left[-\left\|y_{1}\right\|_{L^{\infty}}-1,\left\|y_{1}\right\|_{L^{\infty}}+1\right] .
$$

From the proof of [27], we get the existence of $\mu>0$ such that there exists $z \in Y_{T}$ satisfying

$$
\left\{\begin{array}{l}
z_{t}=z_{x x}+\tilde{f}\left(z+y_{1}\right)-\tilde{f}\left(y_{1}\right) \\
z(t, 0)=0 \\
z(0, x)=y(1 / \varepsilon, x)-y_{1}(x), z(T, x)=0
\end{array}\right.
$$

and the estimate

$$
\|z\|_{Y_{T}} \leq \mu\left\|y(1 / \varepsilon, \cdot)-y_{1}(\cdot)\right\|_{H^{1}(0, L)},
$$

which leads, with $q=z+\tilde{y}_{1}$, to

$$
\left\{\begin{array}{l}
q_{t}=q_{x x}+\tilde{f}(q), \\
q(t, 0)=0 \\
q(0, x)=y(1 / \varepsilon, x), q(T, x)=y_{1}(x),
\end{array}\right.
$$

and

$$
\left\|q-\tilde{y}_{1}\right\|_{Y_{T}} \leq \mu\left\|y(1 / \varepsilon, \cdot)-y_{1}(\cdot)\right\|_{H^{1}(0, L)},
$$

where $\tilde{y}_{1}(t, x):=y_{1}(x)$. From (72) and (73), we get

$$
\left\|q-\tilde{y}_{1}\right\|_{L^{\infty}((0, T) \times(0, L))} \leq 1
$$

for $\left\|y(1 / \varepsilon, \cdot)-y_{1}(\cdot)\right\|_{H^{1}(0, L)}$ small enough. From (71) and (74), we infer that $\tilde{f}(q)=f(q)$, which ends the proof.

\section{Numerical simulations}

Numerical simulations are lead, using Matlab, with the function $f(y)=y^{3}$, that is, we deal with the boundary control system

$$
\left\{\begin{array}{l}
\frac{\partial^{2} y}{\partial t^{2}}=\frac{\partial^{2} y}{\partial x^{2}}+y^{3}, \\
y(t, 0)=0, y_{x}(t, L)=u(t), \\
y(0, \cdot)=y_{0}(\cdot), y_{t}(0, \cdot)=y_{1}(\cdot),
\end{array}\right.
$$

Fix $L=1$. The set $\mathcal{S}$ of steady-states consists of all solutions $y(\cdot)$ of class $C^{2}$ on $[0,1]$ such that

$$
y^{\prime \prime}(x)+y(x)^{3}=0, y(0)=0 .
$$

Since $f$ is odd, this set is connected (see Remark 4). For the numerical simulations, we choose two steady-states $y_{0}$ and $y_{1}$, namely, $y_{0}=0$, and $y_{1}$ denotes the solution of (76) vanishing at $0,1 / 2$ and 


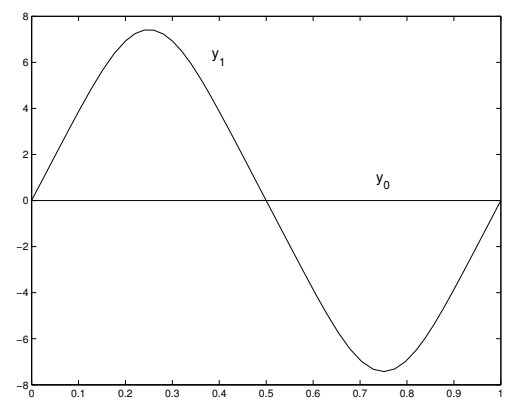

Figure 2: Definition of the steady-states $y_{0}$ and $y_{1}$.

1, and having no other zero on $[0,1]$ (see Fig. 2). Notice that all solutions of (76) can be explicitly computed using elliptic functions.

For every $\tau \in[0,1]$, define the function $\bar{y}(\tau,$.$) on [0,1]$ as the solution of $(76)$ such that

$$
\frac{\partial \bar{y}}{\partial x}(\tau, 0)=\tau y_{1}^{\prime}(0)
$$

and set $\bar{u}(\tau)=\bar{y}(\tau, 1)$. The one-parameter family of linear operators (13) we have to deal with writes

$$
\widetilde{A}(\tau)=\left(\begin{array}{cc}
0 & 1 \\
\triangle+3 \bar{y}(\tau, .)^{2} I d & 0
\end{array}\right)
$$

on the domain $D(\widetilde{A}(\tau))$ given by (14). For $\tau=0$, there holds

$$
\widetilde{A}(0)=\left(\begin{array}{ll}
0 & 1 \\
\triangle & 0
\end{array}\right)
$$

and the eigenvalues and eigenvectors of $\widetilde{A}(0)$ are

$$
\begin{gathered}
\lambda_{k}(0)=\frac{1}{2 L} \ln \frac{\alpha-1}{\alpha+1}+i \frac{k \pi}{L}, \\
e_{k}(0, x)=\frac{1}{A_{k}}\left(\operatorname{sh~} \lambda_{k}(0) x, \lambda_{k}(0) \operatorname{sh} \lambda_{k}(0) x\right),
\end{gathered}
$$

where

$$
A_{k}=\frac{1}{2 L \sqrt{-\operatorname{Re}\left(\lambda_{k}(0)\right)}} \sqrt{\left(\mathrm{e}^{-2 \operatorname{Re}\left(\lambda_{k}(0)\right) L}-\mathrm{e}^{2 \operatorname{Re}\left(\lambda_{k}(0)\right) L}\right)\left(k^{2} \pi^{2}+\left(\operatorname{Re}\left(\lambda_{k}(0)\right)\right)^{2} L^{2}\right)} .
$$

The dual Riesz basis $\left(f_{k}(0, \cdot)\right)_{k \in \mathbb{Z}}$ is given by

$$
f_{k}(0, x)=\frac{A_{k}}{B_{k}}\left(\overline{e_{k}^{1}(0, x)},-\overline{e_{k}^{2}(0, x)}\right),
$$

where

$$
B_{k}=2 \sqrt{-\operatorname{Re}\left(\lambda_{k}(0)\right)} \frac{\left(\operatorname{Re}\left(\lambda_{k}(0)\right) L-i k \pi\right)^{2}}{\sqrt{\left(k^{2} \pi^{2}+\left(\operatorname{Re}\left(\lambda_{k}(0)\right)\right)^{2} L^{2}\right)\left(\mathrm{e}^{-2 \operatorname{Re}\left(\lambda_{k}(0)\right) L}-\mathrm{e}^{2 \operatorname{Re}\left(\lambda_{k}(0)\right) L}\right)}} .
$$

Then, solving by continuation as $\tau \in[0,1]$ boundary value problems, we compute numerically, using a standard finite difference code implemented in Matlab, or a simple shooting method, the first eigenvalues $\lambda_{k}(\tau)$.

On Fig. 3 are represented the eigenvalues $\lambda_{-2}(\tau), \lambda_{-1}(\tau), \lambda_{0}(\tau), \lambda_{1}(\tau), \lambda_{2}(\tau)$, for $\tau \in[0,1]$. Numerically, we choose $L=1$ and $\alpha=1.1$. Then, the eigenvalue $\lambda_{0}(\tau)$ is real, passing from about 
-1.52 when $\tau=0$, to about 9.66 when $\tau=1$. The eigenvalues $\lambda_{1}(\tau)$ and $\lambda_{-1}(\tau)$ are complex and conjugate, up to about $\tau_{0}=0.31$. For $\tau=\tau_{0}$, the eigenvalue $\lambda_{1}\left(\tau_{0}\right)$ is double, and the corresponding eigenspace is of dimension one. For $\tau>\tau_{0}$ both eigenvalues are real, $\lambda_{-1}(\tau)$ is negative, whereas $\lambda_{1}(\tau)$ becomes positive. Finally, if $|k| \geq 2$, the eigenvalue $\lambda_{k}(\tau)$ is algebraically simple, complex, and of negative real part.

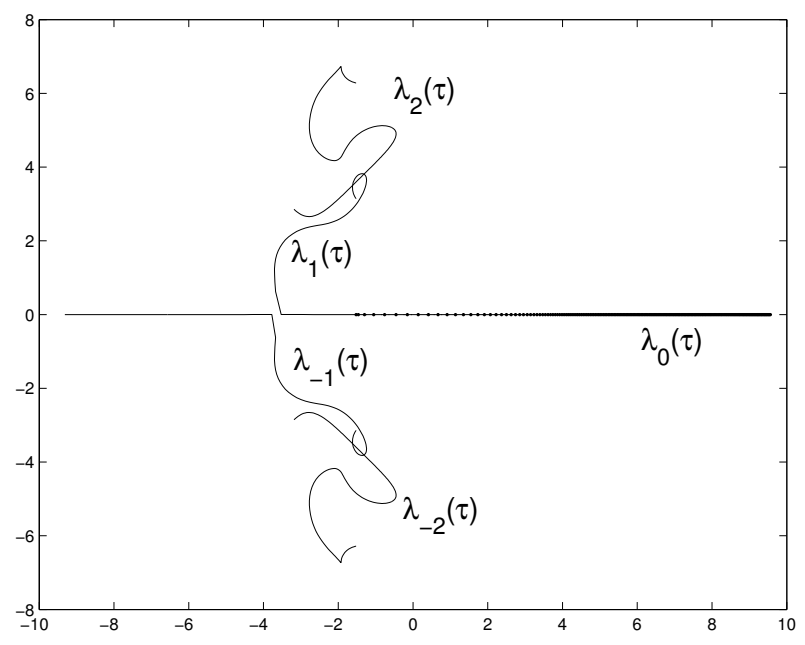

Figure 3: First eigenvalues.

Hence, in this particular case, only the modes corresponding to $\lambda_{0}(\tau)$ and $\lambda_{1}(\tau)$ may become unstable.

From the algorithmic point of view, in order to avoid technical difficulties related to the computation of a Jordan normal form for the matrix $A_{1}(\tau)$ of the finite dimensional system (43), we compute numerically, by continuation, and using a simple shooting method, a basis of the three dimensional real vector space

$$
\operatorname{ker}\left(A_{1}(\tau)-\lambda_{1}(\tau) I\right)\left(A_{1}(\tau)-\lambda_{0}(\tau) I\right)\left(A_{1}(\tau)-\lambda_{-1}(\tau) I\right),
$$

and a dual Riesz basis. Then, we implement a standard pole shifting procedure on this finite dimensional system (see for instance [12]).

Results are drawn on Fig. 4, for $\varepsilon=0.0001$. On the left figure is drawn $y(t, \cdot)$, for $t \in[0,1 / \varepsilon]$; on the right figure is represented $y(1 / \varepsilon, \cdot)-\hat{y}(1 / \varepsilon, \cdot)$. Notice that, if $\varepsilon$ is not small enough, then the solution blows up, as expected (for example, $\varepsilon=0.001$ ).
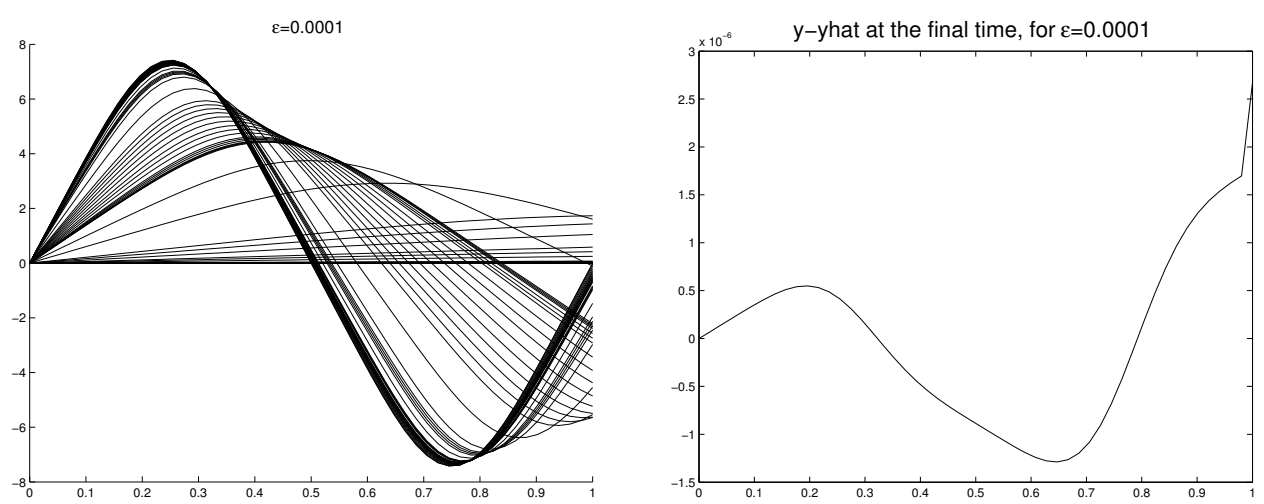

Figure 4: Numerical simulations for $y(t,$.$) , where t \in[0,1 / \varepsilon]$. 


\section{References}

[1] S. A. Avdonin and S. A. Ivanov, Families of exponentials. The method of moments in controllability problems for distributed parameter systems, Cambridge University Press, 1995.

[2] C. Bardos, G. Lebeau and J. Rauch, Sharp sufficient conditions for the observation, control, and stabilization of waves from the boundary, SIAM J. Control Optim., 30, 5 (1992), 1024-1065.

[3] K. Beauchard, Local controllability of a 1-D Schrödinger equation, J. Math. Pures Appl., 84 (2005), 851-956.

[4] P. Cannarsa, V. Komornik and P. Loreti, Well posedness and control of semilinear wave equations with iterated logarithms, ESAIM Cont. Optim. Calc. Var., 4 (1999), 37-56.

[5] T. Cazenave and A. Haraux, Introduction aux problèmes d'évolution semi-linéaires, Math. \& Appl., 1, Ellipses, 1990.

[6] J.-M. Coron, Local controllability of a 1-D tank containing a fluid modeled by the shallow water equations, ESAIM Cont. Optim. Calc. Var., 8 (2002), 513-554.

[7] J.-M. Coron and E. Trélat, Global steady-state controllability of 1-D semilinear heat equations, SIAM J. Control Optim., 43, 2 (2004), 549-569.

[8] J. I. Diaz, Obstruction and some approximate controllability results for the Burgers equation and related problems, Control of partial differential equations and applications, Lecture Notes in Pure and Appl. Math., 174, Dekker, New York, 1996, 63-76.

[9] I. C. Gohberg and M. G. Kreŭn, Introduction to the theory of linear nonselfadjoint operators, Translations of Mathematical Monographs, 18, American Mathematical Society, Providence, R.I., 1969.

[10] B.-Z. Guo, Riesz basis approach to the stabilization of a flexible beam with a tip mass, SIAM J. Control Optim., 39, 6 (2001), 1736-1747.

[11] T. Kato, Perturbation theory for linear operators, Springer-Verlag, New York, 1966.

[12] H. K. Khalil, Nonlinear Systems, Macmillan Publishing Company, New York, Third Edition, 2002.

[13] V. Komornik, Rapid boundary stabilization of linear distributed systems, SIAM J. Control Optim., 35, 5 (1997), 1591-1613.

[14] V. Komornik and E. Zuazua, A direct method for the boundary stabilization of the wave equation, J. Math. Anal. Appl., 69 (1990), 33-54.

[15] H. Kwakernaak and R. Sivan, Linear optimal control systems, Wiley-Interscience, 1972.

[16] L. Li and X. Zhang, Exact controllability for semilinear wave equations, J. Math. Anal. Appl., 250 (2000), 589-597.

[17] J. L. Lions, Exact controllability, stabilization and perturbations for distributed systems, SIAM Rev., 30 (1988), 1-68.

[18] P. Martinez and J. Vancostenoble, Exact controllability in "arbitrarily short time" of the semilinear wave equationn Discrete Contin. Dyn. Syst., 9, 4 (2003), 901-924.

[19] V. P. Mihaŭlov, On Riesz bases in $L^{2}(0,1)$, Dokl. Akad. Nauk SSSR, 144 (1962), 981-984. 
[20] M. Reed and B. Simon, Methods of modern mathematical physics, Vol. 4, Analysis of operators, Academic Press, 1978.

[21] D. L. Russell, Controllability and stabilizability theory for linear partial differential equations: recent progress and open questions, SIAM Rev., 20, 4 (1978), 639-739.

[22] M. Schmidt and E. Trélat, Controllability of Couette-Taylor flows by rotating cylinders, to appear in Comm. Pure Appl. Anal., 2005.

[23] M. A. Shubov, Spectral operators generated by damped hyperbolic equations, Integral Equations Operator Theory, 28, 3 (1997), 358-372.

[24] C.-Z. Xu and G. Sallet, On spectrum and Riesz basis assignment of infinite-dimensional linear systems by bounded linear feedbacks, SIAM J. Cont. Optim., 34, 2 (1996), 521-541.

[25] E. Zuazua, Exact controllability for the semilinear wave equation, J. Math. Pures Appl., 69 (1990), $1-31$.

[26] E. Zuazua, Exact boundary controllability for the semilinear wave equation, Nonlinear partial differential equations and their applications, Collège de France seminar, Vol. X, Paris, 1987-1988, 357-391, Pitman Research Notes Math. Ser., 220, 1991.

[27] E. Zuazua, Exact controllability for semilinear wave equations in one space dimension, Ann. Inst. Henri Poincaré Anal. Non Linéaire, 10, 1 (1993), 109-129. 